\title{
Analyzing Radioligand Binding Data
}

A radioligand is a radioactively labeled drug that can associate with a receptor, transporter, enzyme, or any protein of interest. The term ligand derives from the Latin word ligo, which means to bind or tie. Measuring the rate and extent of binding provides information on the number of binding sites, and their affinity and accessibility for various drugs. While physiological or biochemical measurements of tissue responses to drugs can prove the existence of receptors, only ligand binding studies (or possibly quantitative immunochemical studies) can determine the actual receptor concentration. Radioligand binding experiments are easy to perform, and provide useful data in many fields. For example, radioligand binding studies are used to:

1. Study receptor regulation, for example during development, in diseases, or in response to a drug treatment.

2. Discover new drugs by screening for compounds that compete with high affinity for radioligand binding to a particular receptor.

3. Investigate receptor localization in different organs or regions using autoradiography (UNITS $1.2 \& 1.3$ ).

4. Categorize receptor subtypes.

5. Probe mechanisms of receptor signaling, via measurements of agonist binding and its regulation by ions, nucleotides, and other allosteric modulators.

This unit reviews the theory of receptor binding and explains how to analyze experimental data. Since binding data are usually best analyzed using nonlinear regression, this unit also explains the principles of curve fitting with nonlinear regression. For more general information on analyses of receptor data, see books by Limbird (1996) and Kenakin (1993).

\section{BINDING THEORY}

\section{The Law of Mass Action}

Binding of a ligand to a receptor is a complex process involving conformational changes and multiple noncovalent bonds. The details aren't known in most cases. Despite this complexity, most analyses of radioligand binding experiments successfully use a simple model, called the law of mass action:

$$
\text { ligand }+ \text { receptor } \rightleftarrows \text { ligand } \cdot \text { receptor }
$$

The model is based on these simple ideas:

1. Binding occurs when ligand and receptor collide (due to diffusion) with the correct orientation and sufficient energy. The rate of association (number of binding events per unit of time) equals [ligand] $\times$ [receptor] $\times k_{\text {on }}$, where $k_{\text {on }}$ is the association rate constant in units of $\mathrm{M}^{-1} \mathrm{~min}^{-1}$.

2. Once binding has occurred, the ligand and receptor remain bound together for a random amount of time. The rate of dissociation (number of dissociation events per unit time) equals [ligand $\cdot$ receptor] $\times k_{\text {off }}$, where $k_{\text {off }}$ is the dissociation rate constant expressed in units of $\min ^{-1}$.

3. After dissociation, the ligand and receptor are the same as they were before binding.

Contributed by Harvey Motulsky and Richard Neubig

Current Protocols in Neuroscience (1997) 7.5.1-7.5.55

Copyright (C) 2002 by John Wiley \& Sons, Inc.

Neurochemistry/

Neuropharmacology

\subsection{1}

Supplement 19 


\section{The equilibrium dissociation constant $K_{d}$}

Equilibrium is reached when the rate at which new ligand-receptor complexes are formed equals the rate at which they dissociate:

$$
[\text { ligand }] \times[\text { receptor }] \times k_{\mathrm{on}}=[\text { ligand } \cdot \text { receptor }] \times k_{\mathrm{off}}
$$

Rearrange to define the equilibrium dissociation constant $K_{\mathrm{d}}$.

$$
\frac{[\text { ligand }] \times[\text { receptor }]}{[\text { ligand } \cdot \text { receptor }]}=\frac{k_{\text {off }}}{k_{\text {on }}}=K_{\mathrm{d}}
$$

The $K_{\mathrm{d}}$, expressed in units of moles/liter or molar $(\mathrm{M})$, is the concentration of ligand that occupies half of the receptors at equilibrium. To see this, set [ligand] equal to $K_{\mathrm{d}}$ in the equation above. In this case, [receptor] must equal ligand-receptor, which means that half the receptors are occupied by ligand.

\section{Affinity}

The term affinity is often used loosely. If the $K_{\mathrm{d}}$ is low (e.g., pM or nM), that means that only a low concentration of ligand is required to occupy the receptors, so the affinity is high. If the $K_{\mathrm{d}}$ is larger (e.g., $\mu \mathrm{M}$ or $\mathrm{mM}$ ), a high concentration of ligand is required to occupy receptors, so the affinity is low. The term equilibrium association constant $\left(K_{\mathrm{a}}\right)$ is less commonly used, but is directly related to the affinity of a compound. The $K_{\mathrm{a}}$ is defined to be the reciprocal of the $K_{\mathrm{d}}$, so it is expressed in units of liters/mole. A high $K_{\mathrm{a}}$ (e.g., > $10^{8} \mathrm{M}^{-1}$ ) would represent high affinity.

Because the names sound familiar, it is easy to confuse the equilibrium dissociation constant $\left(K_{\mathrm{d}}\right.$, in molar units) with this dissociation rate constant $\left(K_{\text {off }}\right.$, in $\min ^{-1}$ units), and to confuse the equilibrium association constant $\left(K_{\mathrm{a}}\right.$, in liter/mole units) with the association rate constant ( $K_{\mathrm{on}}$, in $\mathrm{M}^{-1} \mathrm{~min}^{-1}$ units). To help avoid such confusion, equilibrium constants are written as capital " $K$ " and the rate constants with a lower case "k."

A wide range of $K_{\mathrm{d}}$ values are seen with different ligands. Since the $K_{\mathrm{d}}$ equals the ratio $k_{\text {off }} / k_{\text {on }}$, compounds can have different $K_{\mathrm{d}}$ values for a receptor either because the association rate constants are different, the dissociation rate constants are different, or both. In fact, association rate constants are all pretty similar (usually $10^{8}$ to $10^{9} \mathrm{M}^{-1} \mathrm{~min}^{-1}$, which is about two orders of magnitude slower than diffusion), while dissociation rate constants are quite variable (with half-times ranging from seconds to days).

\section{Fractional occupancy at equilibrium}

Fractional occupancy is defined as the fraction of all receptors that are bound to ligand. The law of mass action predicts the fractional receptor occupancy at equilibrium as a function of ligand concentration.

$$
\text { fractional occupancy }=\frac{[\text { ligand } \cdot \text { receptor }]}{[\text { receptor }]_{\text {total }}}=\frac{[\text { ligand } \cdot \text { receptor }]}{[\text { receptor }]+[\text { ligand } \cdot \text { receptor }]}
$$

A bit of algebra creates a useful equation. Multiply both numerator and denominator by [ligand] and divide both by [ligand · receptor]. Then substitute the definition of $K_{\mathrm{d}}$.

$$
\text { fractional occupancy }=\frac{[\text { ligand }]}{[\text { ligand }]+K_{\mathrm{d}}}
$$

Analyzing Radioligand Binding Data

The approach to saturation as [ligand] increases is slower than one might imagine (see Fig. 7.5.1). Even using radioligand at a concentration equal to nine times its $K_{\mathrm{d}}$ will only lead to its binding to $90 \%$ of the receptors. 


\begin{tabular}{|c|c|}
\hline [ligand] $/ K_{\mathrm{d}}$ & $\begin{array}{c}\text { Fractional occupancy } \\
\text { at equilibrium }\end{array}$ \\
\hline 0 & $0 \%$ \\
1 & $50 \%$ \\
4 & $80 \%$ \\
9 & $90 \%$ \\
99 & $99 \%$ \\
\hline
\end{tabular}

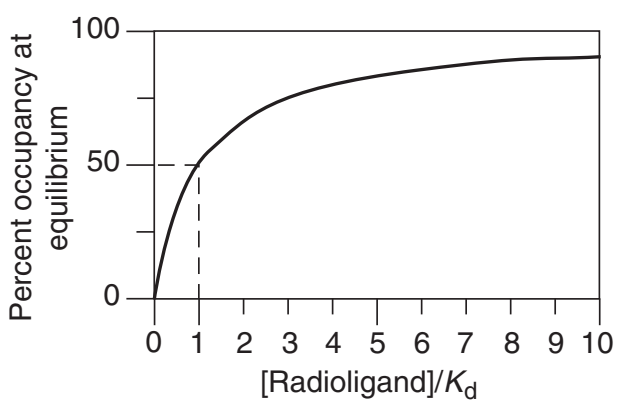

Figure 7.5.1 Occupancy at equilibrium. The fraction of receptors occupied by a ligand at equilibrium depends on the concentration of the ligand compared to its $K_{\mathrm{d}}$.

\section{Assumptions of the law of mass action}

Although termed a "law," the law of mass action is simply a model. It is based on these assumptions:

1. All receptors are equally accessible to ligands.

2. All receptors are either free or bound to ligand. The model ignores any states of partial binding.

3. Neither ligand nor receptor are altered by binding.

4. Binding is reversible.

If these assumptions are not met, there are two choices. One choice is to develop a more complicated model, which is beyond the scope of this unit. The other choice is to analyze the data in the usual way, but interpret the result as an empirical description of the system without attributing rigorous meanings to the $K_{\mathrm{d}}$ and rate constants.

\section{Nonspecific Binding}

In addition to binding to the receptors of physiological interest, radioligands also bind to other (nonreceptor) sites. Binding to the receptor of interest is termed specific binding. Binding to other sites is called nonspecific binding. Because of this operational definition, nonspecific binding can represent several phenomena:

1. The bulk of nonspecific binding represents some sort of interaction of the ligand with membranes. The molecular details are unclear, but nonspecific binding depends on the charge and hydrophobicity of a ligand-but not its exact structure.

2. Nonspecific binding can also result from binding to receptor transporters, or to enzymes not of interest to the investigator (e.g., binding of epinephrine to serotonin receptors).

3. In addition, nonspecific binding can represent binding to the filters used to separate bound from free ligand.

In many systems, nonspecific binding is linear with radioligand concentration. This means that it is possible to account for nonspecific binding mathematically, without ever measuring nonspecific binding directly. To do this, measure only total binding experimentally, and fit the data to models that include both specific and nonspecific components (see More Complicated Situations, below). 
Most investigators, however, prefer to measure nonspecific binding experimentally. To measure nonspecific binding, first block almost all specific binding sites with an unlabeled drug. Under these conditions, the radioligand only binds nonspecifically. This raises two questions: which unlabeled drug should be used and at what concentration?

The most obvious choice of drug to use is the same compound as the radioligand, but unlabeled. In many cases, this is necessary as no other drug is known to bind to the receptors. Most investigators avoid using the same compound as the hot and cold ligand for routine work because both the labeled and unlabeled forms of the drug will bind to the same specific and nonspecific sites. This means that the unlabeled drug will reduce binding purely by isotopic dilution. When possible, it is better to define nonspecific binding with a drug chemically distinct from the radioligand.

The concentration of unlabeled drug should be high enough to block virtually all the specific radioligand binding, but not so much that it will cause more general physical changes to the membrane that might alter specific binding. If studying a well-characterized receptor, a useful rule of thumb is to use the unlabeled compound at a concentration equal to 100 times its $K_{\mathrm{d}}$ for the receptors, or 100 times the highest concentration of radioligand, whichever is higher.

The same results should be obtained from defining nonspecific binding with a range of concentrations of several drugs. Ideally, nonspecific binding is only $10 \%$ to $20 \%$ of the total radioligand binding. If the nonspecific binding makes up more than half of the total binding, it will be hard to get quality data. If the system exhibits a great deal of nonspecific binding, use a different kind of filter, wash with a larger volume of buffer or a different temperature buffer, or use a different radioligand.

\section{Ligand Depletion}

The equations that describe the law of mass action include the variable [ligand], which is the free concentration of ligand. All the analyses presented later in this unit assume that a very small fraction of the ligand binds to receptors (or to nonspecific sites), so that the free concentration of ligand is approximately equal to the concentration added.

In some experimental situations, the receptors are present in high concentration and have a high affinity for the ligand. A large fraction of the radioligand binds to receptors (or nonspecific sites), depleting the amount of ligand remaining free in solution. The discrepancy is not the same in all tubes or at all times. Many investigators use this rule of thumb: if $<10 \%$ of the ligand binds, don't worry about ligand depletion.

If possible, design the experimental protocol to avoid situations where $>10 \%$ of the ligand binds. This can be done by using less tissue in the assays; however, this will also decrease the number of counts. An alternative is to increase the volume of the assay without changing the amount of tissue. In this case, more radioligand will be needed.

If radioligand depletion cannot be avoided, the depletion must be accounted for in the analyses. There are several approaches.

1. Measure the free concentration of ligand in every tube.

2. Calculate the free concentration in each tube by subtracting the number of cpm (counts per minute) of total binding from the cpm of added ligand. This method works only for saturation binding experiments, and cannot be extended to analysis of competition or kinetic experiments. One problem with this approach is that experimental error in determining specific binding also affects the calculated value of free ligand concentration. When fitting curves, both $x$ and $y$ would include experimental 
error, and the errors will be related. This violates the assumptions of nonlinear regression. Using simulated data, Swillens (1995) has shown that this can be a substantial problem. Another problem is that the free concentration of radioligand will not be the same in tubes used for determining total and nonspecific binding. Therefore specific binding cannot be calculated as the difference between the total binding and nonspecific binding.

3. Fit total binding as a function of added ligand using an equation that accounts both for nonspecific binding and for ligand depletion (Swillens, 1995). By analyzing simulated experiments, more reliable results are obtained than those obtained from calculating free ligand by subtraction.

\section{SATURATION BINDING EXPERIMENTS}

Saturation binding experiments determine receptor number and affinity by determining specific binding at various concentrations of the radioligand. Because this kind of experiment can be graphed as a Scatchard plot (more accurately attributed to Rosenthal, 1967), they are sometimes called "Scatchard experiments."

The analyses depend on the assumption that the incubation has reached equilibrium. This can take anywhere from a few minutes to many hours, depending on the ligand, receptor, temperature, and other experimental conditions. Since lower concentrations of radioligand take longer to equilibrate, use a low concentration of radioligand (perhaps $10 \%$ to $20 \%$ of the estimated $K_{\mathrm{d}}$ ) when measuring how long it takes the incubation to reach equilibrium. Experimenters typically use 6 to 12 concentrations of radioligand.

\section{Theory of Saturation Binding}

\section{Nonspecific binding}

Analysis of saturation binding curves requires accounting for nonspecific binding. Although it is possible to account for nonspecific binding mathematically by analyzing total binding (see More Complicated Situations, below), most investigators assess nonspecific binding experimentally by measuring radioligand binding in the presence of a concentration of an unlabeled compound that binds to essentially all the receptors. Since all the receptors are occupied by the unlabeled drug, the radioligand only binds nonspecifically.

Once the nonspecific binding has been determined, subtract it from total binding to calculate specific binding. There are two ways to do this.

1. Experimentally measure nonspecific binding at each concentration of radioligand. Calculate specific binding as total binding minus nonspecific binding at each concentration.

2. An alternative approach relies on the observation that nonspecific binding is generally proportional to the concentration of radioligand (within the concentration range used in the experiment). This means that a graph of nonspecific binding as a function of radioligand binding is generally linear, as shown in Figure 7.5.2. Once nonspecific binding is observed to be linear with radioligand concentration in the system, linear regression can be used to find the best-fit line through the nonspecific binding data. Specific binding is calculated by subtracting the nonspecific binding predicted by that line from the total binding measured at each concentration of radioligand. The major advantage of this approach is that measurements of nonspecific binding at each concentration of radioligand are not needed. For example, one can measure total binding at eight concentrations of radioligand, and nonspecific binding at only four concentrations.

Neurochemistry/ Neuropharmacology

7.5.5 


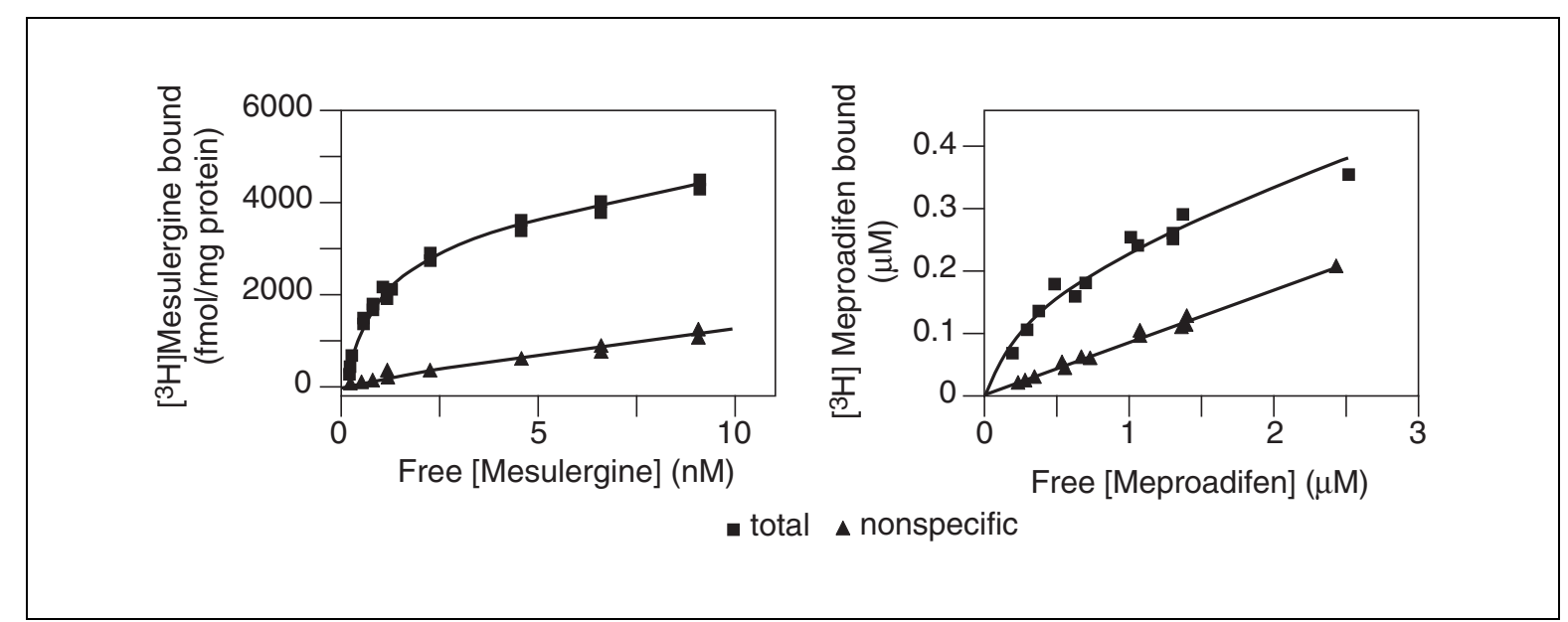

Figure 7.5.2 Examples of nonspecific binding. (A) $\left[{ }^{3} \mathrm{H}\right]$ Mesulergine binding to serotonin receptors has low nonspecific binding ( $<25 \%$ of total binding at the highest concentrations). (B) $\left[{ }^{3} \mathrm{H}\right]$ Meproadifen binding to the ion channel of nicotinic receptors has high nonspecific binding $(>50 \%)$.

Analyzing Radioligand Binding Data

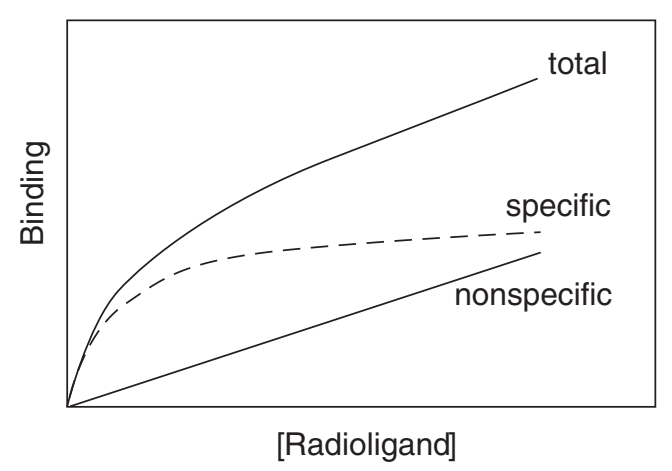

Figure 7.5.3 Total binding, specific binding, and nonspecific binding for a saturation binding experiment.

Panel A of Figure 7.5.2 shows data from a nearly ideal system, where nonspecific binding is less than $25 \%$ of total binding. Panel B shows a less ideal system where nonspecific binding is over $50 \%$ of total binding at high ligand concentrations. If nonspecific binding were much higher than this, it would be very difficult to get reliable results.

\section{Equations used to calculate binding}

Specific binding at equilibrium equals fractional occupancy times the total receptor number $\left(B_{\max }\right)$, and depends on the concentration of radioligand ([L]):

$$
\text { specific binding }=\text { fractional occupancy } \times B_{\max }=\frac{B_{\max } \times[\mathrm{L}]}{K_{\mathrm{d}}+[\mathrm{L}]}
$$

This equation describes a rectangular hyperbola or a binding isotherm. [L] is the concentration of free radioligand, the value plotted on the $x$ axis (see Fig. 7.5.3). $B_{\max }$ is the total number of receptors and is expressed in the same units as the $y$ values (i.e., cpm, sites/cell, or fmol/mg protein). $K_{\mathrm{d}}$ is the equilibrium dissociation constant (expressed in the same units as [L], usually $\mathrm{nM}$ ). Figure 7.5.3 shows the total binding, specific binding, and nonspecific binding for a hypothetical experiment. 
A

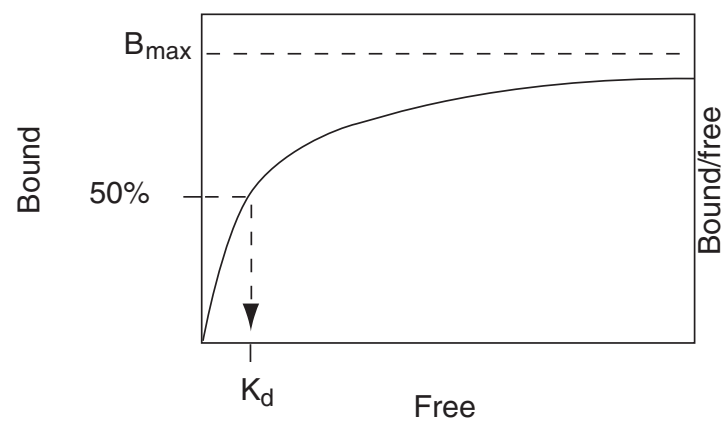

B

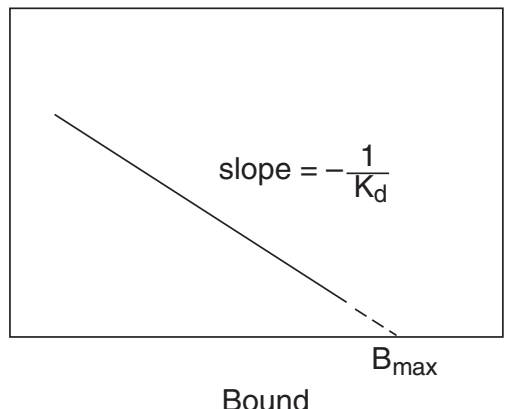

Figure 7.5.4 Displaying results as a Scatchard plot. (A) Specific binding as a function of free radioligand. (B) Transformation of Scatchard data to a plot.

\section{Analysis of Saturation Binding Curves}

\section{Using nonlinear regression to determine $B_{\text {max }}$ and $K_{d}$}

Follow these steps to analyze the data with nonlinear regression:

1. Calculate specific binding at each concentration of ligand (or, in rare cases, decide to analyze only total binding; see More Complicated Situations, below).

2. Convert the specific binding data from counts per minute to more useful units such as $\mathrm{fmol} / \mathrm{mg}$ protein or sites per cell.

3. Define $x$ as the radioligand concentrations in $\mathrm{nM}$ or $\mathrm{pM}$. Define $y$ as the specific binding in $\mathrm{fmol} / \mathrm{mg}$ or sites per cell.

4. Fit the data to this equation.

$$
y=B_{\max } \times x /\left(K_{\mathrm{d}}+x\right)
$$

5. If the curve fitting program does not provide initial values (sometimes called estimated values) automatically, estimate $B_{\max }$ as the largest value of $y$ and estimate $K_{\mathrm{d}}$ as 0.2 times the largest value of $x$.

\section{Are the results reasonable?}

Before accepting the results of the curve fit, ask the questions listed in Table 7.5.1 to determine whether the results are reasonable.

If the results are not reasonable, the experimental protocol may need revision. Also check that the data are being analyzed correctly. In addition, it's possible that the system is more complex than the simple one-site binding model. To determine whether the system follows the assumptions of the simple model, consider the points in Table 7.5.2.

\section{Displaying results as a Scatchard plot}

Before nonlinear regression programs were widely available, scientists transformed data to make a linear graph and then analyzed the transformed data with linear regression. There are several ways to linearize binding data, but Scatchard plots (more accurately attributed to Rosenthal, 1967) are used most often. As shown in Figure 7.5.4, the $x$ axis of the Scatchard plot represents specific binding (usually labeled "bound") and the $y$ axis is the ratio of specific binding to concentration of free radioligand (usually labeled "bound/free"). $B_{\max }$ is the $x$ intercept; $K_{\mathrm{d}}$ is the negative reciprocal of the slope.

Neurochemistry/ Neuropharmacology 
Table 7.5.1 Evaluating the Results of Saturation Binding Curve Analysis

\begin{tabular}{|c|c|}
\hline Question & Comment \\
\hline $\begin{array}{l}\text { Does the calculated curve go near the } \\
\text { data points? }\end{array}$ & $\begin{array}{l}\text { If the curve doesn't go near the data, then } \\
\text { something went wrong with the curve fit, and the } \\
\text { "best-fit" values of } B_{\max } \text { and } K_{\mathrm{d}} \text { should be ignored. }\end{array}$ \\
\hline $\begin{array}{l}\text { Were sufficient concentrations of } \\
\text { radioligand used? }\end{array}$ & $\begin{array}{l}\text { Ideally, the highest concentration should be at least } \\
10 \text { times the } K_{\mathrm{d}} \text {. Calculate the ratio of the highest } \\
\text { radioligand concentration used divided by the } K_{\mathrm{d}} \\
\text { reported by the program (both in } \mathrm{nM} \text { or } \mathrm{pM} \text { ). The } \\
\text { ratio should be greater than } 10 .\end{array}$ \\
\hline Is the $B_{\max }$ reasonable? & $\begin{array}{l}\text { Typical values for } B_{\max } \text { are } 10 \text { to } 1000 \mathrm{fmol} \text { binding } \\
\text { sites per milligram of membrane protein, } 1000 \text { sites } \\
\text { per cell, or } 1 \text { receptor per square micron of } \\
\text { membrane. If using cells transfected with receptor } \\
\text { genes, then the } B_{\max } \text { may be } 10 \text { to } 100 \text { times larger } \\
\text { than these values. }\end{array}$ \\
\hline Is the $K_{\mathrm{d}}$ reasonable? & $\begin{array}{l}\text { Typical values for } K_{\mathrm{d}} \text { of useful radioligands range } \\
\text { between } 10 \mathrm{pM} \text { and } 100 \mathrm{nM} \text {. If the } K_{\mathrm{d}} \text { is much } \\
\text { lower than } 10 \mathrm{pM} \text {, the dissociation rate is probably } \\
\text { very slow and it will be difficult to achieve } \\
\text { equilibrium. If the } K_{\mathrm{d}} \text { is much higher than } 100 \mathrm{nM} \text {, } \\
\text { the dissociation rate will probably be fast, and may } \\
\text { result in the loss of binding sites during separation } \\
\text { of bound from free radioligand. }\end{array}$ \\
\hline $\begin{array}{l}\text { Are the standard errors too large? Are } \\
\text { the confidence intervals too wide? }\end{array}$ & $\begin{array}{l}\text { Nonlinear regression programs report the } \\
\text { uncertainty of the best-fit values for } B_{\max } \text { and } K_{\mathrm{d}} \text { as } \\
\text { standard errors and } 95 \% \text { confidence intervals. } \\
\text { Divide the SE of the } B_{\max } \text { by the } B_{\max } \text {, and divide } \\
\text { the SE of the } K_{\mathrm{d}} \text { by the } K_{\mathrm{d}} \text {. If either ratio is much } \\
\text { larger than } \sim 20 \% \text {, look further to determine why. }\end{array}$ \\
\hline Is the nonspecific binding too high? & $\begin{array}{l}\text { Divide the nonspecific binding at the highest } \\
\text { concentration of radioligand by the total binding at } \\
\text { that concentration. Nonspecific binding should } \\
\text { usually be less than } 50 \% \text { of the total binding. }\end{array}$ \\
\hline
\end{tabular}

Analyzing Radioligand Binding Data
If the curve doesn't go near the data, then something went wrong with the curve fit, and the "best-fit" values of $B_{\max }$ and $K_{\mathrm{d}}$ should be ignored. radioligand concentration used divided by the $K_{\mathrm{d}}$ reported by the program (both in $\mathrm{nM}$ or $\mathrm{pM}$ ). The sites per milligram of membrane protein, 1000 sites per cell, or 1 receptor per square micron of membrane. If using cells transfected with receptor genes, then the $B_{\max }$ may be 10 to 100 times larger than these values. between $10 \mathrm{pM}$ and $100 \mathrm{nM}$. If the $K_{\mathrm{d}}$ is much lower than $10 \mathrm{pM}$, the dissociation rate is probably very slow and it will be difficult to achieve equilibrium. If the $K_{\mathrm{d}}$ is much higher than $100 \mathrm{nM}$, of bound from free radioligand. standard errors and $95 \%$ confidence intervals. Divide the $\mathrm{SE}$ of the $B_{\max }$ by the $B_{\max }$, and divide the SE of the $K_{\mathrm{d}}$ by the $K_{\mathrm{d}}$. If either ratio is much larger than $\sim 20 \%$, look further to determine why.

Divide the nonspecific binding at the highest that concentration. Nonspecific binding should usually be less than $50 \%$ of the total binding.

Table 7.5.2 Evaluating the Assumptions of Saturation Binding Analysis

\begin{tabular}{|c|c|}
\hline Assumption & Comment \\
\hline Binding has reached equilibrium. & $\begin{array}{l}\text { It takes longest for the lower concentrations to } \\
\text { equilibrate, so test equilibration time with the } \\
\text { lowest concentration of radioligand. }\end{array}$ \\
\hline $\begin{array}{l}\text { There is only one population of } \\
\text { receptors. }\end{array}$ & $\begin{array}{l}\text { See Theory: Comparing One- and Two-Site Models, } \\
\text { below. }\end{array}$ \\
\hline $\begin{array}{l}\text { Only a small fraction of the radioligand } \\
\text { binds, therefore the free concentration } \\
\text { is essentially identical to the } \\
\text { concentration added. }\end{array}$ & $\begin{array}{l}\text { Compare the cpm obtained for total binding to the } \\
\text { amount of ligand. If the ratio is greater than } 10 \% \text { at } \\
\text { any concentration, this assumption has been } \\
\text { violated. Increase the volume of the reaction but use } \\
\text { the same amount of tissue. }\end{array}$ \\
\hline $\begin{array}{l}\text { There is no cooperativity. Binding of a } \\
\text { ligand to one binding site does not alter } \\
\text { the affinity of another binding site. }\end{array}$ & See Cooperativity, below. \\
\hline
\end{tabular}


A

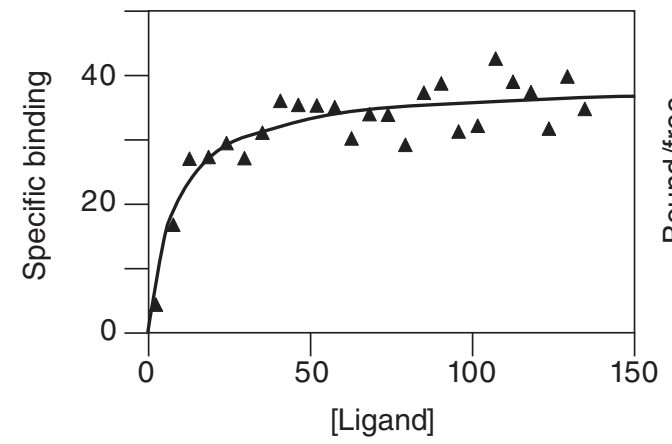

B

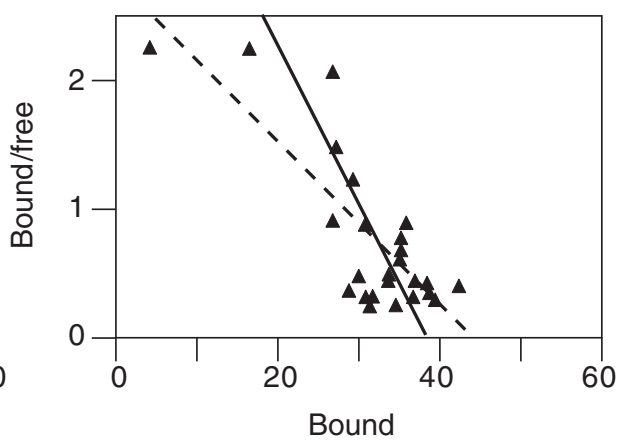

Figure 7.5.5 Why Scatchard plots (though useful for displaying data) should not be used for analyzing data. (A) Experimental data with best-fit curve determined by nonlinear regression. (B) Scatchard plot of the data. The solid line corresponds to the $B_{\max }$ and $K_{\mathrm{d}}$ determined by nonlinear regression in panel A. The dashed line was determined by linear regression of transformed data in panel $B$. The results of linear regression of the Scatchard plot are not the most accurate values for $B_{\max }\left(x\right.$ intercept) or $K_{\mathrm{d}}$ (negative reciprocal of the slope).

When making a Scatchard plot, there are two ways to express the $y$ axis.

1. One choice is to express both free ligand and specific binding in cpm so the ratio bound/free is a unitless fraction. The advantage of this choice is that you can interpret $y$ values as the fraction of radioligand bound to receptors. If the highest $y$ value is large $(>0.10)$, then the free concentration of radioligand will be substantially less than the added concentration, and the standard analyses will yield inaccurate values for $B_{\max }$ and $K_{\mathrm{d}}$. In this situation, either revise the experimental protocol or use special analysis methods that deal with ligand, as discussed previously. The disadvantage of this choice of units is that the slope of the line cannot be interpreted without performing unit conversions.

2. An alternative is to express the $y$ axis as the ratio of units used to display bound and free on the saturation binding graph (i.e., sites $/$ cell $/ \mathrm{nM}$ or $\mathrm{fmol} / \mathrm{mg} / \mathrm{nM}$ ). While these values are hard to interpret, they simplify calculation of the $K_{\mathrm{d}}$, which equals the negative reciprocal of the slope. The specific binding units cancel when calculating the slope. The negative reciprocal of the slope is expressed in units of concentration (nM) which equals the $K_{\mathrm{d}}$.

\section{The problem with using Scatchard plots to analyze saturation binding experiments}

While Scatchard plots are very useful for visualizing data, they are not the most accurate way to analyze data. The problem is that the linear transformation distorts the experimental error. Linear regression assumes that the scatter of points around the line follows a Gaussian distribution and that the standard deviation is the same at every value of $x$. These assumptions are not true with the transformed data. A second problem is that the Scatchard transformation alters the relationship between $x$ and $y$. The value of $x$ (bound) is used to calculate $y$ (bound/free), and this violates the assumptions of linear regression.

Since these assumptions are violated, the $B_{\max }$ and $K_{\mathrm{d}}$ values determined by linear regression of Scatchard-transformed data are likely to be further from the actual values than the $B_{\max }$ and $K_{\mathrm{d}}$ determined by nonlinear regression. Nonlinear regression produces the most accurate results, whereas a Scatchard plot produces only approximate results.

Figure 7.5.5 illustrates the problem of transforming data. The left panel shows data that follows a rectangular hyperbola (binding isotherm). The solid curve was determined by

Neurochemistry/ Neuropharmacology

7.5.9 


\begin{tabular}{|c|rr|rc|}
\hline $\begin{array}{c}\text { [Radioligand] } \\
(\mathrm{nM})\end{array}$ & \multicolumn{2}{|c|}{$\begin{array}{c}\text { Total binding } \\
(\mathrm{cpm})\end{array}$} & $\begin{array}{c}\text { Nonspecific binding } \\
(\mathrm{cpm})\end{array}$ \\
\hline 0.125 & 818 & 826 & & 88 \\
0.25 & 1856 & 1727 & 94 & \\
0.5 & 3452 & 3349 & & 375 \\
1.0 & 6681 & 6055 & 354 & \\
2.0 & 10077 & 9333 & & 1525 \\
4.0 & 13715 & 13277 & 1573 & \\
\hline
\end{tabular}

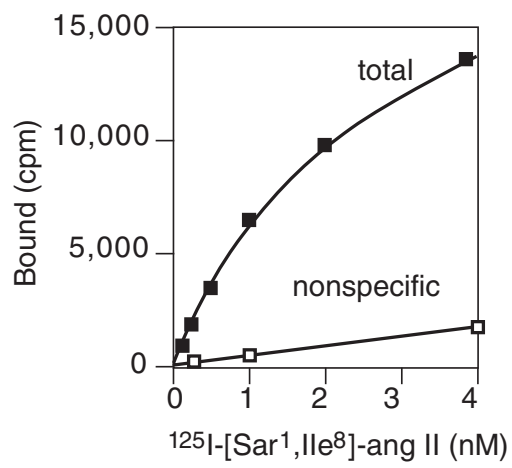

Figure 7.5.6 Sample saturation binding experiment. The ligand binding to angiotensin receptors in a membrane preparation was measured. Total and nonspecific binding are shown.

nonlinear regression. The right panel is a Scatchard plot of the same data. The solid line shows how that same curve would look after a Scatchard transformation. The dotted line shows the linear regression fit of the transformed data. The transformation amplified and distorted the scatter, and thus the linear regression fit does not yield the most accurate values for $B_{\max }$ and $K_{\mathrm{d}}$. In this example, the $B_{\max }$ determined by the Scatchard plot is $\sim 25 \%$ too large and the $K_{\mathrm{d}}$ determined by the Scatchard plot is too high. The errors could just as easily have gone in the other direction.

The experiment in Figure 7.5.5 was designed to determine the $B_{\max }$ with little concern for the value of $K_{\mathrm{d}}$. Therefore, it was appropriate to obtain only a few data points at the beginning of the curve and many in the plateau region. Note however how the Scatchard transformation gives undo weight to the data point collected at the lowest concentration of radioligand (the lower left point in panel A, the upper left point in panel B). This point dominates the linear regression calculations on the Scatchard graph. It has "pulled" the regression line to become shallower, resulting in an overestimate of the $B_{\max }$.

Again, although it is inappropriate to analyze data by performing linear regression on a Scatchard plot, it is often helpful to display data as a Scatchard plot. Many people find it easier to visually interpret Scatchard plots than binding curves, especially when comparing results from different experimental treatments.

\section{Example of a Saturation Binding Experiment}

\section{Raw data}

Figure 7.5.6 shows duplicate values for total binding of six concentrations of a radioligand to angiotensin receptors on membranes of cells transfected with an angiotensin gene ( $R$. Neubig, unpub. observ.). The figure also shows nonspecific binding (assessed with $10 \mu \mathrm{M}$ unlabeled angiotensin II) at three concentrations of radioligand.

\section{Calculating specific binding}

Since nonspecific binding was only determined at three concentrations of radioligand, the standard method of subtracting each nonspecific value from the corresponding total value cannot be used. Instead, the fact (confirmed in other experiments) that nonspecific binding is proportional to radioligand concentration is relied upon, and the best-fit value of nonspecific binding is subtracted from each total binding value. This can be done in

Analyzing Radioligand Binding Data one step by choosing "remove baseline analysis" in GraphPad Prism software. Alternatively: 
Table 7.5.3 Calculating Specific Binding

\begin{tabular}{|c|c|c|c|c|c|c|c|}
\hline \multirow{2}{*}{$\begin{array}{l}\text { [Radio- } \\
\text { ligand] } \\
(\mathrm{nM})\end{array}$} & \multicolumn{2}{|c|}{ Total binding (cpm) } & \multirow{2}{*}{$\begin{array}{c}\text { Computed } \\
\text { nonspecific } \\
\text { binding }(\mathrm{cpm})\end{array}$} & \multicolumn{2}{|c|}{$\begin{array}{l}\text { Calculated specific } \\
\text { binding }(\mathrm{cpm})\end{array}$} & \multicolumn{2}{|c|}{$\begin{array}{l}\text { Specific binding } \\
\quad(\mathrm{fmol} / \mathrm{mg})\end{array}$} \\
\hline & Duplicate 1 & Duplicate 2 & & Duplicate 1 & Duplicate 2 & Duplicate 1 & Duplicate 2 \\
\hline 0.125 & 818 & 826 & 34 & 784 & 792 & 17.9 & 18.1 \\
\hline 0.25 & 1856 & 1727 & 82 & 1774 & 1645 & 40.5 & 37.6 \\
\hline 0.5 & 3452 & 3349 & 180 & 3272 & 3169 & 74.8 & 72.4 \\
\hline 1.0 & 6681 & 6055 & 375 & 6306 & 5680 & 144.1 & 129.8 \\
\hline 2.0 & 10077 & 9333 & 766 & 9311 & 8567 & 212.8 & 195.8 \\
\hline 4.0 & 13715 & 13277 & 1547 & 12168 & 11730 & 278.1 & 268.1 \\
\hline
\end{tabular}

1. Use linear regression. The best fit line through the nonspecific binding data is:

nonspecific binding in $\mathrm{cpm}=-15.25+390.5([$ radioligand $]$ in $\mathrm{nM})$

2. Use this equation to calculate nonspecific binding at each of the six radioligand concentrations.

3. Subtract that calculated value from the observed total binding to compute specific binding (Table 7.5.3).

\section{Converting units}

Convert from cpm to $\mathrm{fmol} / \mathrm{mg}$ using the amount of protein in each tube $(0.01 \mathrm{mg})$, the efficiency of the counting (90\%), and the specific radioactivity of the ligand (2190 $\mathrm{Ci} / \mathrm{mmole})$.

$$
\mathrm{fmol} / \mathrm{mg}=\frac{\mathrm{cpm}}{2.22 \times 10^{12} \mathrm{dpm} / \mathrm{Ci} \times 0.90 \mathrm{cpm} / \mathrm{dpm} \times 2190 \mathrm{Ci} / \mathrm{mmol} \times 10^{-12} \mathrm{mmol} / \mathrm{fmol} \times 0.01 \mathrm{mg}}
$$

NOTE:

1. Receptors in membrane preparations are often expressed as fmol of receptor per milligram of membrane protein. One fmol is $10^{-15}$ moles.

2. Counting efficiency is the fraction of the radioactive disintegrations that are detected by the counter. This example uses a radioligand labeled with ${ }^{125} \mathrm{I}$, so the efficiency $(90 \%)$ is very high.

3. The Curie (C:) is a unit of radioactivity and equals $2.22 \times 10^{12}$ radioactive disintegrations per minute.

4. The value $2190 \mathrm{Ci} / \mathrm{mmole}$ is worth remembering. It is the specific activity of ligands iodinated with ${ }^{125} \mathrm{I}$, when every molecule is labeled with one iodine.

5. Simplifying the equation, simply divide the cpm by 43.756 (see Table 7.5.3).

\section{Fitting a curve to determine $B_{\text {max }}$ and $K_{d}$}

When fitting the example data to a curve, one must decide whether to enter the data as six points or twelve. Entering each replicate individually is better, as it provides more data to the curve fitting procedure. This should be avoided only when the replicates are not independent (i.e., when experimental error in one value is likely to affect the other value as well). In this case each replicate was determined in a separate tube poured over a separate filter, and all the data were obtained from one membrane preparation. Except 


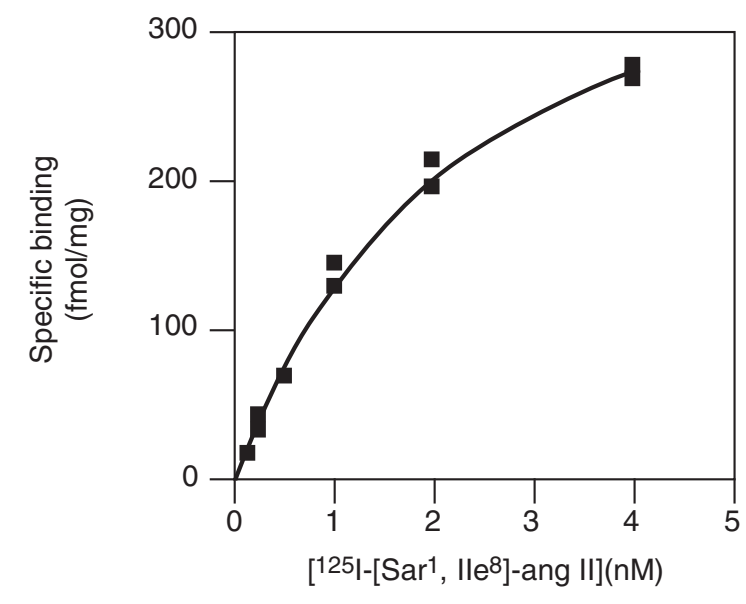

Figure 7.5.7 Specific binding with the best-fit curve determined by nonlinear regression. These data are the same as those shown in Figure 7.5.6 and Table 7.5.3.

for errors in preparing the radioligand dilutions, experimental errors will affect each value independently.

Follow these steps to fit the data.

1. If the data-fitting program understands the concept of duplicates, then enter the data with radioligand concentration as six $x$ values and the duplicate values of specific binding at each concentration. If the program does not understand how to deal with duplicates, enter each concentration value twice in the $x$ column, to fill twelve rows. Enter the specific binding data as a column of twelve $y$ values.

2. Choose nonlinear regression and choose or enter the one-site binding equation. Expressed in the format of most curve fitting systems, it is:

$$
y=\left(B_{\max } \times x\right) /\left(K_{\mathrm{d}}+x\right)
$$

3. If the chosen nonlinear regression program does not provide initial values automatically, estimate values for $B_{\max }$ and $K_{\mathrm{d}}$. For $B_{\max }$, enter a value a bit higher than the highest value in the data, perhaps 300 for this example. For $K_{\mathrm{d}}$, estimate the concentration of radioligand that binds to half the sites, perhaps $0.5 \mathrm{nM}$. These estimated values do not have to be very accurate.

4. Start the curve fit, and note the results. The best-fit value of $B_{\max }$ is 429. It is expressed in the same units as the $y$ values entered (fmol/mg). The best-fit value of $K_{\mathrm{d}}$ is 2.27 . It is expressed in the same units as the $x$ values entered (nM).

5. Graph the specific binding with the best-fit curve as shown in Figure 7.5.7.

\section{Creating a Scatchard plot}

A Scatchard plot is a graph of specific binding vs. the ratio of specific binding to free radioligand. For specific binding, the two replicates are averaged (individual replicates could have been shown). For the example in Figure 7.5.8, bound/free is expressed as fmol/mg divided by $\mathrm{nM}$.

Analyzing Radioligand Binding Data
Figure 7.5.8 shows the Scatchard transformation of the specific binding data. Since it is not appropriate to determine the $K_{\mathrm{d}}$ and $B_{\max }$ from linear regression of a Scatchard plot, derive the solid line on the graph from the best-fit values using nonlinear regression: 
Specific binding Bound/free (fmol/mg protein) $\mathrm{fmol} / \mathrm{mg} / \mathrm{nM}$

\begin{tabular}{|r|r|}
\hline 18 & 143.5 \\
39 & 155.5 \\
73 & 146.5 \\
136 & 136.3 \\
203 & 101.7 \\
272 & 68.0 \\
\hline
\end{tabular}

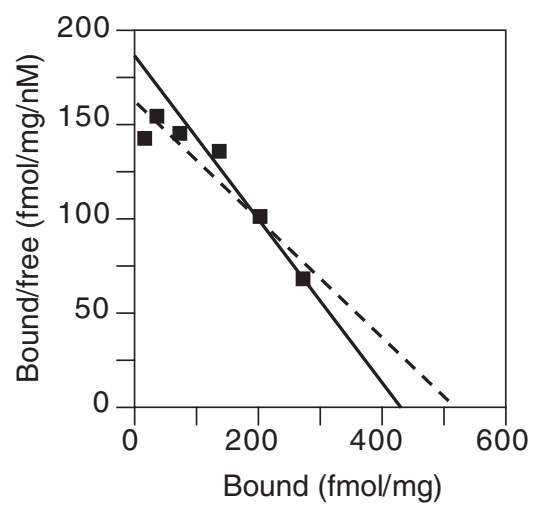

Figure 7.5.8 Scatchard transformation of the data from Figure 7.5.7. The solid line was created (as explained in the text) from the best-fit values of $B_{\max }$ and $K_{\mathrm{d}}$ determined from nonlinear regression. This is the correct line to show on a Scatchard plot. The dashed line was determined by linear regression of the Scatchard transformed data. It is shown here for comparison only; it is not informative or helpful.

1. The $x$ intercept of the Scatchard plot is $B_{\max }$, which equals 429 by nonlinear regression, so one end of the line is at $x=429, y=0$.

2. The slope of the line is the negative reciprocal of the $K_{\mathrm{d}}$. Since the $K_{\mathrm{d}}$ is $2.27 \mathrm{nM}$, the slope must be $-1 / 2.27$, which equals $-0.4405 \mathrm{nM}^{-1}$.

3. The $y$ intercept divided by the $x$ intercept equals the negative slope. We know the slope and the $x$ intercept, so can derive the $y$ intercept. It equals -slope $\times x$ intercept $=0.4405 \times 429=188.1$.

4. Draw the line from $x=0, y=188.1$ to $x=429, y=0$, as in Figure 7.5.8.

Figure 7.5.8 also shows the dotted line derived by linear regression of the Scatchard transformed data. This is shown only to emphasize the difference between it and the best-fit line derived from nonlinear regression. The linear regression line should not be used for data analysis and does not aid data presentation.

\section{Critiquing the experiment}

This example is not an ideal experiment. Consider these points:

The highest concentration of radioligand used $(4 \mathrm{nM})$ is not even twice the $K_{\mathrm{d}}(2.27 \mathrm{nM})$. Ideally the highest concentration of radioligand should be ten times the $K_{\mathrm{d}}$. In addition, the specific binding of the first few points lies below the best fit curve. There are many possible explanations for this, including chance, but it may be because the system is not at equilibrium. The lowest concentrations take the longest to equilibrate, so it is possible that the first few concentrations had not equilibrated, resulting in an underestimate of specific binding at equilibrium.

\section{More Complicated Situations}

\section{Measuring total binding only}

Rather than measure both total and nonspecific binding at each concentration of ligand, measure only total binding. Then fit the data to the equation below, which defines total binding as a function of ligand concentration. Total binding is the sum of specific binding and nonspecific binding. Fit the data to this equation to find best-fit values of $B_{\max }, K_{\mathrm{d}}$,

Neurochemistry/ Neuropharmacology

7.5.13 
and NS (the slope of the graph of nonspecific binding as a function of radioligand concentration):

$$
\text { total binding }=\text { specific binding }+ \text { nonspecific binding }=\frac{B_{\max } \times[\mathrm{L}]}{K_{\mathrm{d}}+[\mathrm{L}]}+\mathrm{NS} \times[\mathrm{L}]
$$

This approach assumes that nonspecific binding is proportional to [ligand]. This assumption is reasonable if the nonspecific binding is due to general binding to membranes, but may not be reasonable if some of the nonspecific binding represents binding to receptors or transporters other than the one being studied.

To get useful results with this approach requires high-quality data and at least ten data points, including some well above the ligand $K_{\mathrm{d}}$.

\section{Two classes of binding sites}

If the radioligand binds to two classes of binding sites, the specific binding data can be fit to this equation:

$$
\text { specific binding }=\frac{B_{\max 1} \times[\mathrm{L}]}{K_{\mathrm{d} 1}+[\mathrm{L}]}+\frac{B_{\max 2} \times[\mathrm{L}]}{K_{\mathrm{d} 2}+[\mathrm{L}]}
$$

This equation assumes that the radioligand binds to two independent noninteracting binding sites, and that the binding to each site follows the law of mass action. A comparison of the one-site and two-site fits will be addressed later in this unit (see Theory: Comparing One- and Two-Site Models, below).

Meaningful results will be obtained from a two-site fit only if you have ten or more data points spaced over a wide range of radioligand concentrations. Binding should be measured at radioligand concentrations below the high-affinity $K_{\mathrm{d}}$ and above the low-affinity $K_{\mathrm{d}}$.

\section{Homologous competitive binding curves}

Some investigators determine the $K_{\mathrm{d}}$ and $B_{\max }$ of a ligand by holding the concentration of the radioligand constant and competing with various concentrations of the unlabeled ligand. This approach will be discussed below.

\section{COMPETITIVE BINDING EXPERIMENTS}

\section{Theory of Competitive Binding}

\section{Using competitive binding curves}

Competitive binding experiments measure the binding of a single concentration of labeled ligand in the presence of various concentrations (often twelve to sixteen) of unlabeled ligand. Competitive binding experiments are used to:

1. Validate an assay. Perform competitive binding experiments with a series of drugs whose potencies at the receptor of interest are known from functional experiments. Demonstrating that these drugs bind with the expected potencies, or at least the expected order of potency, helps prove that the radioligand has identified the correct receptor. This kind of experiment is crucial, because there is usually no point studying a binding site unless it has physiological significance.

2. Determine whether a drug binds to the receptor. Thousands of compounds can be screened to find drugs that bind to the receptor. This can be faster and easier than other screening methods. 
3. Investigate the interaction of low affinity drugs with receptors. Binding assays are only useful when the radioligand has a high affinity $\left(K_{\mathrm{d}}<100 \mathrm{nM}\right.$ or so $)$. A radioligand with low affinity generally has a fast dissociation rate constant, and so won't stay bound to the receptor while washing the filters. To study the binding of a low affinity drug, use it as an unlabeled competitor.

4. Determine receptor number and affinity by using the same compound as the labeled and unlabeled ligand (see Homologous Competitive Binding Curves, below).

\section{Performing the experiment}

Competitive binding experiments use a single concentration of radioligand and require incubation until equilibrium is reached. That raises two questions: how much radioligand should be used, and how long does it take to equilibrate?

There is no clear answer to the first question. Higher concentrations of radioligand result in higher counts and thus lower counting error, but these experiments are more expensive and have higher nonspecific binding. Lower concentrations save money and reduce nonspecific binding but result in fewer counts from specific binding and thus more counting error. Many investigators choose a concentration approximately equal to the $K_{\mathrm{d}}$ of the radioligand for binding to the receptor, but this is not universal. In general, you should aim for a minimum of $1000 \mathrm{cpm}$ from specific binding in the absence of competitor.

Many investigators' first thoughts are that binding will reach equilibration in the time it takes the radioligand to reach equilibrium in the absence of competitor. It turns out that this may not be long enough. Incubations should last four to five times the half-life for receptor dissociation as determined in a dissociation experiment.

\section{Equations for competitive binding}

Competitive binding curves are described by this equation:

$$
\text { total radioligand binding }=\mathrm{NS}+\frac{(\text { total }-\mathrm{NS})}{1+10^{\log [\mathrm{D}]-\log \left(\mathrm{IC}_{50}\right)}}
$$

The $x$ axis of Figure 7.5.9 shows varying concentrations of unlabeled drug on a log scale. The $y$ axis can be expressed as cpm or converted to more useful units like fmol bound per milligram protein or number of binding sites per cell. Some investigators like to normalize the data from $100 \%$ (no competitor) to $0 \%$ (nonspecific binding at maximal concentrations of competitor).

The top of the curve shows a plateau at the amount of radioligand bound in the absence of the competing unlabeled drug. This equals the parameter total in the equation. The bottom of the curve is a plateau equal to nonspecific binding; this is nonspecific (NS) in the equation. These values are expressed in the units of the $y$ axis. The difference between the top and bottom plateaus is the specific binding. Note that this not the same as $B_{\max }$. When using a low concentration of radioligand (to save money and avoid nonspecific binding), only a fraction of receptors will be bound (even in the absence of competitor), so specific binding will be much lower than the $B_{\max }$.

The concentration of unlabeled drug that results in radioligand binding halfway between the upper and lower plateaus is called the $\mathrm{IC}_{50}$ (inhibitory concentration $50 \%$ ), also called the $\mathrm{EC}_{50}$ (effective concentration 50\%). The $\mathrm{IC}_{50}$ is the concentration of unlabeled drug that blocks half the specific binding, and it is determined by three factors:

1. The $K_{\mathrm{i}}$ of the receptor for the competing drug. This is what is to be determined. It is the equilibrium dissociation constant for binding of the unlabeled drug - the concen-

Neurochemistry/ Neuropharmacology

7.5.15 


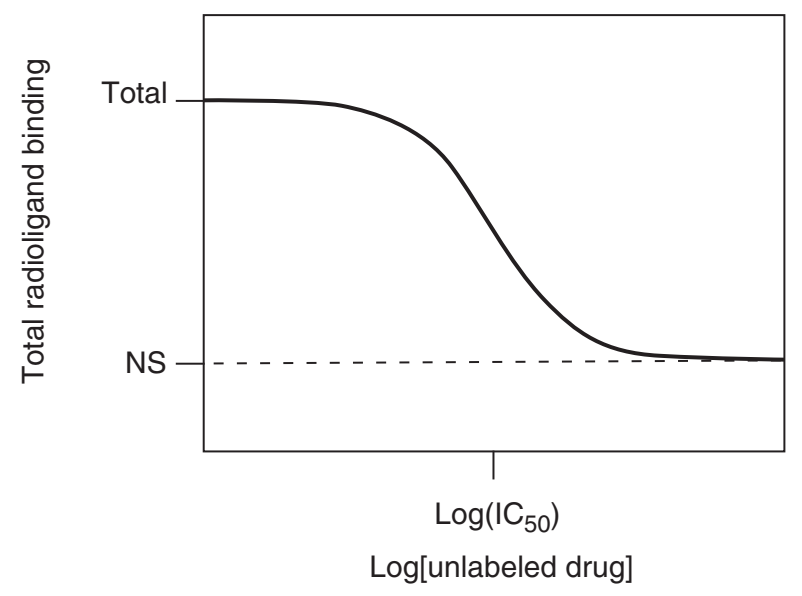

Figure 7.5.9 Schematic of a competitive binding experiment.

tration of the unlabeled drug that will bind to half the binding sites at equilibrium in the absence of radioligand or other competitors. The $K_{\mathrm{i}}$ is proportional to the $\mathrm{IC}_{50}$. If the $K_{\mathrm{i}}$ is low (i.e., the affinity is high), the $\mathrm{IC}_{50}$ will also be low.

2. The concentration of the radioligand. If a higher concentration of radioligand is used, it will take a larger concentration of unlabeled drug to compete for the binding. Therefore, increasing the concentration of radioligand will increase the $\mathrm{IC}_{50}$ without changing the $K_{\mathrm{i}}$.

3. The affinity of the radioligand for the receptor $\left(K_{\mathrm{d}}\right)$. It takes more unlabeled drug to compete for a tightly bound radioligand (small $K_{\mathrm{d}}$ ) than for a loosely bound radioligand (high $K_{\mathrm{d}}$ ). Using a radioligand with a smaller $K_{\mathrm{d}}$ (higher affinity) will increase the $\mathrm{IC}_{50}$.

Calculate the $K_{\mathrm{i}}$ from the $\mathrm{IC}_{50}$, using the equation of Cheng and Prusoff (1973).

$$
K_{\mathrm{i}}=\frac{\mathrm{IC}_{50}}{1+\frac{[\text { radioligand }]}{K_{d}}}
$$

Remember that $K_{\mathrm{i}}$ is a property of the receptor and unlabeled drug, while $\mathrm{IC}_{50}$ is a property of the experiment. By changing experimental conditions (changing the radioligand used or changing its concentration), the $\mathrm{IC}_{50}$ will change without affecting the $K_{\mathrm{i}}$.

This equation is based on the following assumptions:

1. Only a small fraction of either the labeled or unlabeled ligand has bound. This means that the free concentration is virtually the same as the added concentration.

2. The receptors are homogeneous and all have the same affinity for the ligands.

3. There is no cooperativity-binding to one binding site does not alter affinity at another site.

4. The experiment has reached equilibrium.

5. Binding is reversible and follows the law of mass action.

Analyzing

6. The $K_{\mathrm{d}}$ of the radioligand is known from an experiment performed under similar conditions. 


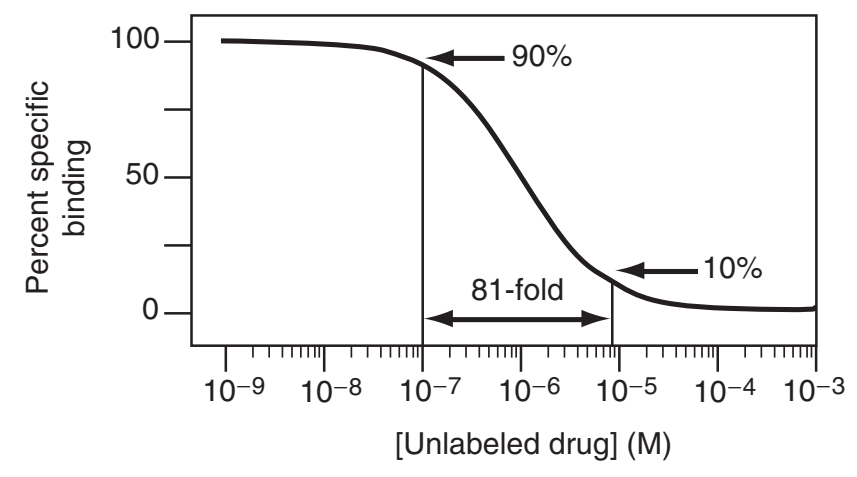

Figure 7.5.10 Steepness of a competitive binding curve. This graph shows the results at equilibrium when radioligand and competitor bind to the same binding site. The curve will descend from $90 \%$ binding to $10 \%$ binding over an 81 -fold increase in competitor concentration.

If the labeled and unlabeled ligand compete for a single binding site, the steepness of the competitive binding curve is determined by the law of mass action (see Fig. 7.5.10). The curve descends from $90 \%$ specific binding to $10 \%$ specific binding with an 81 -fold increase in the concentration of the unlabeled drug. More simply, nearly the entire curve will cover two log units (100-fold change in concentration).

\section{Analyzing Competitive Binding Data}

\section{Using nonlinear regression to determine the $K_{i}$}

Follow these steps to determine the $K_{\mathrm{i}}$ with nonlinear regression.

1. Enter the $x$ values as the logarithm of the concentration of unlabeled compound, or enter the concentrations, and use the program to convert to logarithms. Since $\log (0)$ is undefined, the log scale cannot accommodate a concentration of zero. Instead enter a very low concentration. For example, if the lowest concentration of unlabeled compound is $10^{-10} \mathrm{M}$, then enter -12 for the zero concentration.

2. Enter the $y$ values as cpm total binding. There is little advantage to converting to units such as fmol/mg or sites/cell. There is also little advantage to converting to percent specific binding.

3. Select the competitive binding equation (TOP is binding in the absence of competitor, BOTTOM is binding at maximal concentrations of competitor, $\log \mathrm{IC}_{50}$ is the logarithm base 10 of the $\mathrm{IC}_{50}$ ):

$$
y=\mathrm{NS}+\frac{\mathrm{TOTAL}-\mathrm{NS}}{1+10^{x-\log \mathrm{IC}_{50}}}
$$

4. If the chosen nonlinear regression program doesn't provide initial estimates automatically, enter these values. For NS, enter the smallest $y$ value. For TOTAL, enter the largest $y$ value. For $\log \left(\mathrm{IC}_{50}\right)$, enter the average of the smallest and largest $x$ values.

5. If the data do not form clear plateaus at the top and bottom of the curve, consider fixing top or bottom to constant values. TOTAL can be fixed to the binding measured in the absence of competitor and NS to binding measured in the presence of a large concentration of a standard drug known to block radioligand binding to essentially all receptors.

Neurochemistry/ Neuropharmacology

7.5.17 
6. Start the curve fitting to determine TOTAL, NS, and $\log \left(\mathrm{IC}_{50}\right)$.

7. Calculate the $\mathrm{IC}_{50}$ as the antilog of $\log \left(\mathrm{IC}_{50}\right)$.

8. Calculate the $K_{\mathrm{i}}$ using this equation:

$$
K_{\mathrm{i}}=\frac{\mathrm{IC}_{50}}{1+\frac{[\text { radioligand }]}{K_{d}}}
$$

\section{When to set total and NS constant}

In order to determine the best-fit value of $\mathrm{IC}_{50}$, the nonlinear regression program must be able to determine the $100 \%$ (total) and $0 \%$ (nonspecific) plateaus. If there is data over a wide range of concentrations of unlabeled drug, the curve will have clearly defined bottom and top plateaus and the program should have no trouble fitting all three values (both plateaus and the $\mathrm{IC}_{50}$ ).

With some experiments, the competition data may not define a clear bottom plateau. If data are fit in the usual way, the program might stop with an error message, or it might find a nonsense value for the nonspecific plateau (it might even be negative). If the bottom plateau is incorrect, the $\mathrm{IC}_{50}$ will also be incorrect. To solve this problem, define the nonspecific binding from other data. All drugs that bind to the same receptor should compete for all specific radioligand binding and reach the same bottom plateau value. When running the curve fitting program, set the bottom plateau of the curve (NS) to a constant equal to binding in the presence of a standard drug known to block all specific binding.

Similarly, if the curve doesn't have a clear top plateau, set the total binding to be a constant equal to binding in the absence of any competitor.

\section{Interpreting the Results of Competitive Binding Curves}

\section{Are the results reasonable?}

Table 7.5.4 presents some questions to consider when determining if the results are reasonable and logical.

Do the data follow the assumptions of the analysis?

Table 7.5.5 lists the assumptions.

Table 7.5.4 Evaluation the Results of Competitive Binding Curve Analyses

Question Comment

Is the $\log \left(\mathrm{IC}_{50}\right)$ reasonable?

Are the standard errors too large? Are the confidence intervals too wide?

Are the values of TOTAL and NS reasonable?
The $\mathrm{IC}_{50}$ should be near the middle of the curve, with at least several concentrations of unlabeled competitor on either side of it.

The SE of the $\log \left(\mathrm{IC}_{50}\right)$ should be $<0.5 \log$ unit (ideally much less).

TOTAL should be near the binding observed in the absence of competitor. NS should be near the binding observed in the presence of a maximal concentration of competitor. If the best-fit value of NS is negative, consider fixing it to a constant value equal to nonspecific binding. 
Table 7.5.5 Evaluating the Assumptions of Competitive Binding Analyses

\begin{tabular}{|c|c|}
\hline Assumption & Comment \\
\hline Binding has reached equilibrium. & $\begin{array}{l}\text { Competitive binding incubations take longer to } \\
\text { incubate than saturation binding incubations. } \\
\text { Incubate for } 4 \text { to } 5 \text { times the half life for radioligand } \\
\text { dissociation. }\end{array}$ \\
\hline $\begin{array}{l}\text { There is only one population of } \\
\text { receptors }\end{array}$ & See Theory: Comparing One- and Two-Site Models. \\
\hline $\begin{array}{l}\text { Only a small fraction of the radioligand } \\
\text { binds, therefore the free concentration } \\
\text { is essentially identical to the } \\
\text { concentration added. }\end{array}$ & $\begin{array}{l}\text { Compare the total binding in the absence of } \\
\text { competitor in cpm, to the amount of ligand added in } \\
\text { cpm. If the ratio is }>10 \% \text { at any concentration, then } \\
\text { you've violated this assumption. }\end{array}$ \\
\hline $\begin{array}{l}\text { There is no cooperativity. Binding of a } \\
\text { ligand to one binding site does not alter } \\
\text { the affinity of another binding site. }\end{array}$ & See Cooperativity. \\
\hline
\end{tabular}

\section{Why determine $\log \left(I_{50}\right)$ rather than $I C_{50}$ ?}

The equation for a competitive binding curve (see Theory of Competitive Binding, Equations for competitive binding, above) looks a bit strange since it combines logarithms and antilogarithms (10 to the power). A bit of algebra simplifies it:

$$
y=\text { nonspecific }+\frac{(\text { total }- \text { nonspecific })}{1+\frac{[\mathrm{drug}]}{\mathrm{IC}_{50}}}
$$

Fitting data to this equation results in the same best-fit curve and the same $\mathrm{IC}_{50}$. However, the confidence interval for the $\mathrm{IC}_{50}$ will be different.

Which confidence interval is correct? With nonlinear regression, the standard errors of the variables are only approximately correct. Since the confidence intervals are calculated from the standard errors, they too are only approximately correct. The problem is that the real confidence interval may not be symmetrical around the best-fit value. It may extend further in one direction than the other. However, nonlinear regression programs always calculate symmetrical confidence intervals (unless you use advanced techniques). Therefore, when writing the equation for nonlinear regression, choose variables so the uncertainty is as symmetrical as possible. Because data are collected at concentrations of unlabeled drug equally spaced on a log axis, the uncertainty is symmetrical when the equation is written in terms of the $\log \left(\mathrm{IC}_{50}\right)$, but is not symmetrical when written in terms of $\mathrm{IC}_{50}$. Confidence intervals are more accurate when the equation is written in terms of the $\log \left(\mathrm{IC}_{50}\right)$.

Figure 7.5.11 (R. Neubig, unpub. observ.) shows competition of unlabeled yohimbine for labeled UK14341 (an $\alpha_{2}$ adrenergic agonist).

1. Enter the data into a nonlinear regression program. Enter the logarithm of concentration of the unlabeled ligand in the $x$ column, and the triplicate values of total binding in the $x$ columns. If the selected program does not allow entry of triplicate values, enter each $\log$ of concentration three times.

2. Fit the data to a one-site competitive binding curve. If necessary, enter it in this format:

$$
y=\mathrm{NS}+\left[\text { Total }-\frac{\mathrm{NS}}{1+10^{\left(x-\log \mathrm{IC}_{50}\right)}}\right]
$$

Neurochemistry/ Neuropharmacology

7.5.19 


\begin{tabular}{|c|lll|}
\hline $\begin{array}{c}\text { Log } \\
\text { [competitor](M) }\end{array}$ & \multicolumn{3}{|c|}{$\begin{array}{c}\text { Binding (cpm) } \\
\text { in triplicate }\end{array}$} \\
\hline-12.0 & 4549 & 4380 & 4554 \\
-10.0 & 4604 & 4803 & 4213 \\
-9.5 & 4353 & 4278 & 4508 \\
-9.0 & 4192 & 4156 & 3972 \\
-8.5 & 4053 & 4420 & 4191 \\
-8.0 & 3453 & 3018 & 3024 \\
-7.5 & 2587 & 2946 & 2367 \\
-7.0 & 1295 & 1405 & 1402 \\
-6.5 & 886 & 880 & 888 \\
-6.0 & 591 & 612 & 603 \\
-5.5 & 580 & 559 & 555 \\
-5.0 & 521 & 545 & 555 \\
\hline
\end{tabular}

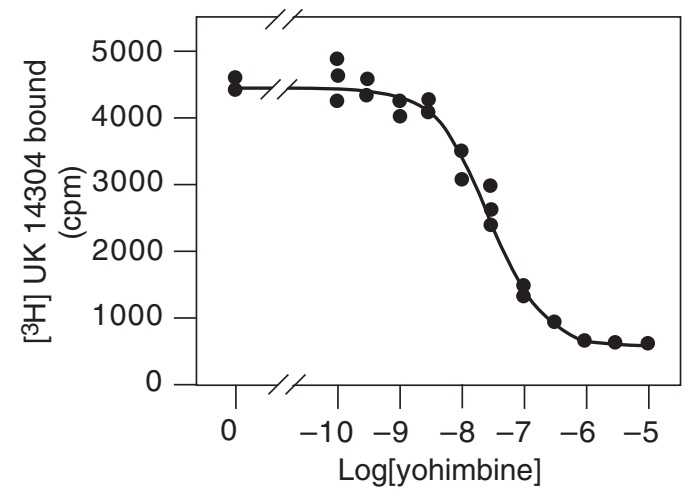

Figure 7.5.11 Example of a competitive binding experiment. Yohimbine competes for radioligand binding to $\alpha_{2}$ receptors on membranes.

3. If the nonlinear regression program does not provide initial values automatically, estimate the values of the variables. TOTAL is the top plateau of the curve, so estimate its value from the highest data values, perhaps 4500. NS is the bottom plateau, so estimate its value from the lowest data values, perhaps 500. $\log \left(\mathrm{IC}_{50}\right)$ is the $x$ value in the middle of the curve. From looking at the data, estimate its value as -7 . None of these estimates has to be very accurate, and the nonlinear regression will probably work fine even if the estimates are fairly different than the values listed here.

4. Note the best-fit results: $\mathrm{NS}=530.3$, TOTAL $=4418$, and $\log \left(\mathrm{IC}_{50}\right)=-7.532$.

5. Convert the $\log \left(\mathrm{IC}_{50}\right)$ to the $\mathrm{IC}_{50}$ by taking the antilog. $\mathrm{IC}_{50}=29.4 \mathrm{nM}$.

6. Convert the $\mathrm{IC}_{50}$ to $K_{\mathrm{i}}$. To do this, the concentration of radioligand used ( $\left.2.0 \mathrm{nM}\right)$ and its $K_{\mathrm{d}}$ for the receptors $(0.88 \mathrm{nM}$, determined in a separate saturation binding experiment not shown here) must be known.

$$
K_{\mathrm{i}}=\frac{\mathrm{IC}_{50}}{1+\frac{[\text { radioligand }]}{K_{\mathrm{d}}}}=\frac{29.4 \mathrm{nM}}{1+\frac{2.0 \mathrm{nM}}{0.88 \mathrm{nM}}}=8.98 \mathrm{nM}
$$

\section{Homologous Competitive Binding Curves}

A competitive binding experiment is termed homologous when the same compound is used as the hot and cold ligand. The term heterologous is used when the hot and cold ligands differ. Homologous competitive binding experiments can be used to determine the affinity of a ligand for the receptor and the receptor number. In other words, the experiment has the same goals as a saturation binding curve. Because homologous competitive binding experiments use a single concentration of radioligand (which can be low), they consume less radioligand and thus are more practical when radioligands are expensive or difficult to synthesize.

Analyzing Radioligand Binding Data
To analyze a homologous competitive binding curve, the following assumptions must be made: 
1. The receptor has identical affinity for the labeled and unlabeled ligand. If you choose an iodinated radioligand, you should also use an iodinated unlabeled compound (using nonradioactive iodine), because iodination often changes the binding properties of ligands.

2. There is no cooperativity.

3. There is no ligand depletion. The methods in this section assume that only a small fraction of ligand binds. In other words, the method assumes that free concentration equals the concentration added.

4. There is only one class of binding sites. It is difficult to detect a second class of binding sites unless the number of lower affinity sites vastly exceeds the number of higher affinity receptors (because the single low concentration of radioligand used in the experiment will bind to only a small fraction of low affinity receptors).

Analyze a homologous competitive binding curve using the same equation used for a one-site heterologous competitive binding to determine the top and bottom plateaus and the $\mathrm{IC}_{50}$.

The Cheng and Prusoff equation (see Theory of Competitive Binding, above) can be used to calculate the $K_{\mathrm{i}}$ from the $\mathrm{IC}_{50}$. In the case of a homologous competitive binding experiment, assume that the hot and cold ligand have identical affinities so that $K_{\mathrm{d}}$ and $K_{\mathrm{i}}$ are the same. An algebraic rearrangement yields:

$$
K_{\mathrm{d}}=K_{\mathrm{i}}=\mathrm{IC}_{50}-[\text { radioligand }]
$$

Set the concentration of radioligand in the experimental design, and determine the $\mathrm{IC}_{50}$ from nonlinear regression. The difference between the two is the $K_{\mathrm{d}}$ of the ligand (assuming hot and cold ligands bind the same).

The difference between the top and bottom plateaus of the curve represents the specific binding of radioligand at the concentration used. Depending on how much radioligand is used, this value may be close to the $B_{\max }$ or far from it. To determine the $B_{\max }$, divide the specific binding by the fractional occupancy, calculated from the $K_{\mathrm{d}}$ and the concentration of radioligand.

$$
B_{\max }=\frac{\text { TOP }- \text { BOTTOM }}{\text { fractional occupancy }}=\frac{\text { TOP }- \text { BOTTOM }}{\left.\frac{[\text { radioligand }]}{\left(K_{\mathrm{d}}+[\text { radioligand }]\right.}\right)}
$$

\section{Example of homologous competitive binding}

Figure 7.5.12 shows data from a binding experiment using $\left[{ }^{3} \mathrm{H}\right]$ yohimbine to quantify $\alpha_{2}$ adrenergic receptors to compete with unlabeled yohimbine. Since there is no reason to think that the tritium label will alter yohimbine's affinity for the receptor, the method from the previous section can be used to quantify receptor number and affinity.

1. Enter the data into a nonlinear regression program. Enter the logarithm of concentration as $x$ and cpm bound as $y$. The first point represents a control with no yohimbine. Since the log of zero is undefined, this cannot be shown on a log scale. Instead enter this value as -12 (the exact value is a bit arbitrary).

2. Fit the data using nonlinear regression to a sigmoidal equation; or, for manual entry, enter it like this:

$$
y=\text { BOTTOM }+\frac{(\text { TOP }- \text { BOTTOM })}{1+10^{\left(x-\log \mathrm{IC}_{50}\right)}}
$$

Neurochemistry/ Neuropharmacology

7.5.21

Supplement 26 


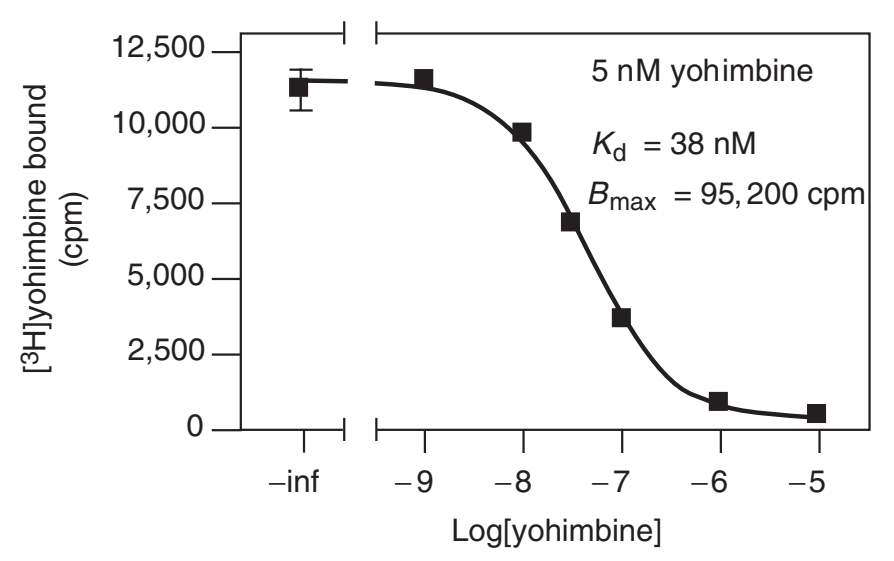

Figure 7.5.12 Example of homologous competitive binding experiment. The hot and cold ligands are identical.

3. Note the best-fit results: $\mathrm{BOTTOM}=350.6$; $\mathrm{TOP}=11,510 ; \log \left(\mathrm{IC}_{50}\right)=-7.370$; and $\mathrm{IC}_{50}=42.6 \mathrm{nM}$.

4. Compute the $K_{\mathrm{i}}$ as the difference between the $\mathrm{IC}_{50}$ and the amount of radioligand added to all tubes. $K_{\mathrm{i}}=42.6 \mathrm{nM}-5.0 \mathrm{nM}=37.6 \mathrm{nM}$.

5. Calculate the fraction of the receptors occupied by the $5 \mathrm{nM}\left[{ }^{3} \mathrm{H}\right]$ yohimbine used in the experiment. Fractional occupancy $=[$ ligand $] /\left([\right.$ ligand $\left.]+K_{\mathrm{d}}\right)=5 /(5.0+37.6)=$ $11.73 \%$.

6. Calculate the specific binding of $5 \mathrm{nM}$ radioligand as the difference between the top and bottom plateaus. Specific binding $=11,510-350=11,160 \mathrm{cpm}$.

7. Divide the specific binding by the fractional occupancy to determine the total number of binding sites, $B_{\max }$. It equals $11,160 / 0.1173=95,140 \mathrm{cpm}$.

8. Finally convert to more useful units. In this example there were $6 \times 10^{4}$ cells per well, the specific activity of the $\left[{ }^{3} \mathrm{H}\right]$ yohimbine was $78 \mathrm{Ci} / \mathrm{mmole}$, and the scintillation counting efficiency was $33 \%$. Calculate receptors/cell using the equation:

$$
\begin{aligned}
& \frac{95,140 \mathrm{cpm} \times 6.02 \times 10^{20} \text { receptors } / \mathrm{mmol}}{2.22 \times 10^{12} \mathrm{dpm} / \mathrm{Ci} \times 0.33 \mathrm{cpm} / \mathrm{dpm} \times 78 \mathrm{Ci} / \mathrm{mmol} \times 60,000 \text { cells }} \\
& =1.67 \times 10^{7} \mathrm{receptors} / \text { cell }
\end{aligned}
$$

\section{The Slope Factor or Hill Slope}

Many competitive binding curves are shallower than predicted by the law of mass action for binding to a single site. The steepness of a binding curve can be quantified with a slope factor, often called a Hill slope. A one-site competitive binding curve that follows the law of mass action has a slope of -1.0 . If the curve is more shallow, the slope factor will be a negative fraction (i.e., -0.85 or -0.60 ; see Fig. 7.5.13). The slope factor is negative because the curve goes downhill.

To quantify the steepness of a competitive binding curve (or a dose-response curve), fit the data to this equation:

$$
\text { total radioligand binding }=\mathrm{NS}+\frac{(\text { Total }-\mathrm{NS})}{1+10^{\left(\log \mathrm{IC}_{50}-\log [\mathrm{D}]\right) \times \text { slope factor }}}
$$




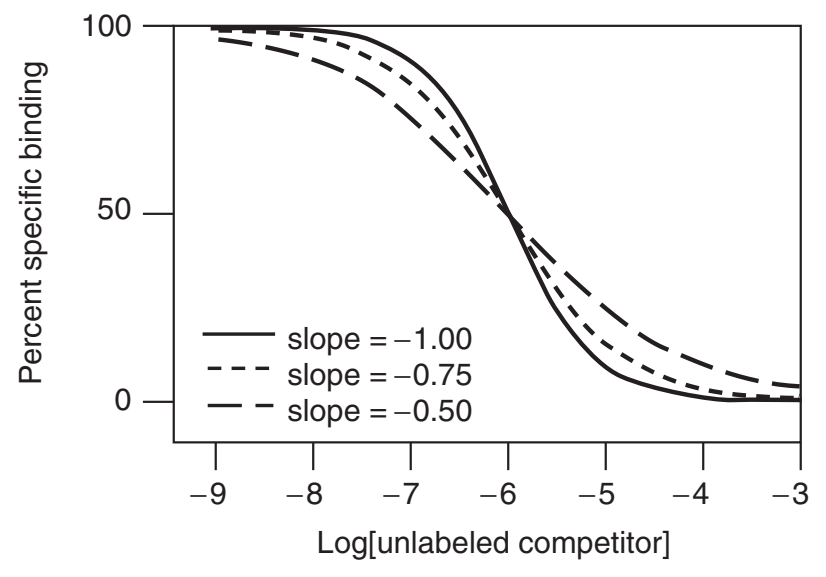

Figure 7.5.13 Examples of slope factors. The slope factor quantifies the steepness of the curve, and is determined by nonlinear regression of competitive binding data. It is not the same as the slope of the curves at the midpoints.

The slope factor is a number that describes the steepness of the curve. In most situations, there is no way to interpret the value in terms of chemistry or biology. If the slope factor differs significantly from -1.0 , then the binding does not follow the law of mass action with a single site.

Explanations for shallow binding curves include:

1. Heterogeneous receptors. The receptors do not all bind the unlabeled drug with the same affinity. This can be due to the presence of different receptor subtypes, or due to heterogeneity in receptor coupling to other molecules such as G proteins. In Fig. 7.5.12, the slope factor equals -0.78 .

2. Negative cooperativity. Binding sites are clustered (perhaps several binding sites per molecule) and binding of the unlabeled ligand to one site causes the remaining site(s) to bind the unlabeled ligand with lower affinity.

3. Curve fitting problems. If the top and bottom plateaus are not correct, then the slope factor is not meaningful. Don't try to interpret the slope factor unless the curve has clear top and bottom plateaus.

\section{KINETIC BINDING EXPERIMENTS}

\section{Theory of Binding, Kinetic}

\section{Dissociation experiments}

A dissociation binding experiment measures the "off rate" of radioligand dissociating from the receptor. Perform dissociation experiments to fully characterize the interaction of ligand and receptor and confirm that the law of mass action applies. They may also be used to help design the experimental protocol. If the dissociation is fast, filter and wash the samples quickly so that negligible dissociation occurs. It may also require lowering the temperature of the buffer used to wash the filters, or switching to a centrifugation or dialysis assay. If the dissociation is slow, then the samples can be filtered at a more leisurely pace, because the dissociation will be negligible during the wash. 
To perform a dissociation experiment, first allow ligand and receptor to bind, perhaps to equilibrium. At that point, block further binding of radioligand to receptor using one of these methods:

1. If the tissue is attached to a surface, remove the buffer containing radioligand and replace with fresh buffer without radioligand.

2. Spin the suspension and resuspend in fresh buffer.

3. Add a very high concentration of an unlabeled ligand (perhaps 100 times its $K_{\mathrm{i}}$ for that receptor). It will instantly bind to nearly all the unoccupied receptors and block binding of the radioligand.

4. Dilute the incubation by a large factor, perhaps a 20- to 100-fold dilution. This will reduce the concentration of radioligand by that factor. At such a low concentration, new binding of radioligand will be negligible. This method is only practical when using a fairly low radioligand concentration so its concentration after dilution is far below its $K_{\mathrm{d}}$ for binding.

5. After initiating dissociation, measure binding over time (typically 10 to 20 measurements) to determine how rapidly the ligand dissociates from the receptors.

The law of mass action predicts that dissociation of radioligands from receptors follows this equation:

$$
\text { total binding }=\mathrm{NS}+(\text { Total }-\mathrm{NS}) \times e^{-k_{\mathrm{off}} t}
$$

Total binding and nonspecific binding (NS) are expressed in cpm, fmol/mg protein, or sites/cell. Time $(t)$ is usually expressed in minutes. The dissociation rate constant $\left(k_{\text {off }}\right)$ is expressed in units of inverse time, usually $\mathrm{min}^{-1}$. Since it is hard to think in those units, it helps to calculate the half-life for dissociation, which equals $\ln (2) / k_{\text {off }}$ or $0.6931 / k_{\text {off }}$. In one half-life, half the radioligand will have dissociated (see Fig. 7.5.14). In two half-lives, three quarters of the radioligand will have dissociated, etc.

Typically the dissociation rate constant of useful radioligands is between 0.001 and 0.1 $\mathrm{min}^{-1}$. If the dissociation rate constant is any faster, it would be difficult to perform radioligand binding experiments as the radioligand would dissociate from the receptors while you wash the filters. If the dissociation rate constant is any slower, it would be hard to reach equilibrium.

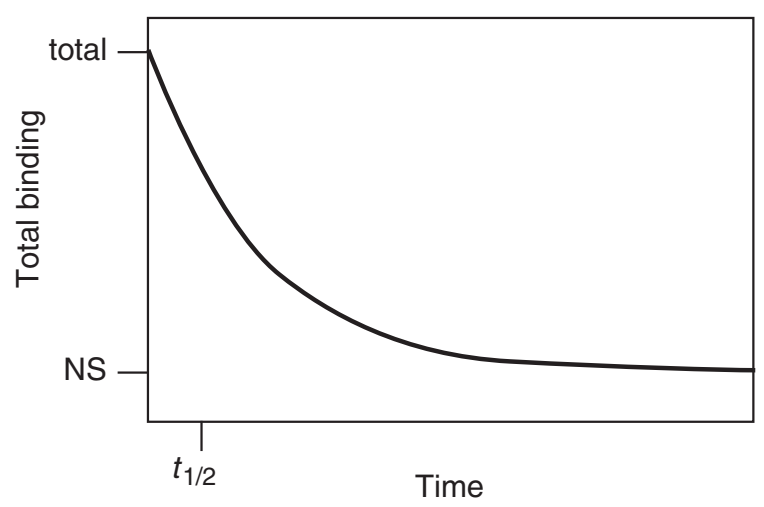

Figure 7.5.14 Schematic of a dissociation kinetic experiment. 


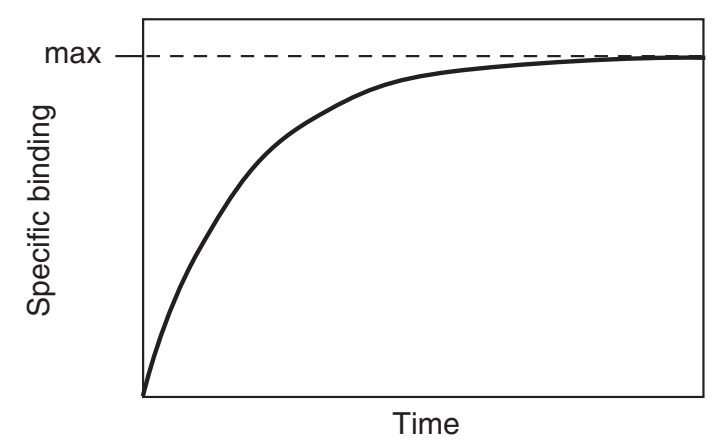

Figure 7.5.15 Schematic of an association kinetic experiment.

\section{Association binding experiments}

Association binding experiments are used to determine the association rate constant. This value is useful to characterize the interaction of the ligand with the receptor. It also is important as it permits the determination of how long it takes to reach equilibrium in saturation and competition experiments.

To perform an association experiment, add a single concentration of radioligand and measure specific binding at various times thereafter.

Association of ligand to receptors (according to the law of mass action) follows this equation:

$$
\text { specific binding }=\operatorname{Max} \times\left(1-e^{-k_{\mathrm{ob}} \times t}\right)
$$

In Figure 7.5.15, note that the maximum binding (Max) is not the same as $B_{\max }$. The maximum (equilibrium) binding achieved in an association experiment depends on the concentration of radioligand. Low to moderate concentrations of radioligand will bind to only a small fraction of all the receptors no matter how long binding is allowed to proceed.

Note that the equation used for fitting does not include the association rate constant, $k_{\mathrm{on}}$, but rather contains the observed rate constant, $k_{\mathrm{ob}}$, which is expressed in units of inverse time (usually $\min ^{-1}$ ). The $k_{\mathrm{ob}}$ is a measure of how quickly the incubation reaches equilibrium, and in the case of a simple bimolecular binding reaction is defined by this equation:

$$
k_{\mathrm{ob}}=k_{\mathrm{off}}+k_{\mathrm{on}} \times[\text { radioligand }]
$$

The equation defines $k_{\mathrm{ob}}$ as a function of three factors:

1. The association rate constant, $k_{\mathrm{on}}$. This is what is to be determined. If $k_{\mathrm{on}}$ is larger (faster), $k_{\mathrm{ob}}$ will be larger as well.

2. The concentration of radioligand. When using more radioligand, the system will equilibrate faster and $k_{\mathrm{ob}}$ will be larger.

3. The dissociation rate constant, $k_{\text {off }}$. It may be surprising to discover that the observed rate of association depends in part on the dissociation rate constant. This makes sense because an association experiment doesn't directly measure how long it takes radioligand to bind, but rather measures how long it takes the binding to reach equilibrium. Equilibrium is reached when the rate of the forward binding reaction

Neurochemistry/ Neuropharmacology

7.5.25 
equals the rate of the reverse dissociation reaction. If the radioligand dissociates quickly from the receptor, equilibrium will be reached faster, but there will be less binding at equilibrium. If the radioligand dissociates slowly, equilibrium will be reached more slowly and there will be more binding at equilibrium.

To calculate the association rate constant, usually expressed in units of $\mathrm{M}^{-1} \mathrm{~min}^{-1}$, use the following equation. Typically ligands have association rate constants of $\sim 10^{8} \mathrm{M}^{-1} \mathrm{~min}^{-1}$.

$$
k_{\mathrm{on}}=\frac{k_{\mathrm{ob}}-k_{\mathrm{off}}}{[\text { radioligand }]}
$$

\section{Analyzing Association Binding Data}

\section{Using nonlinear regression to determine $\boldsymbol{k}_{\text {off }}$}

1. Enter the $x$ data as time in minutes.

2. Enter the $y$ data as total binding in cpm.

3. Choose the exponential dissociation equation. It may be expressed as:

$$
\mathrm{y}=\operatorname{Span} \times \exp (-\mathrm{K} \times x)+\text { Plateau }
$$

4. If the chosen nonlinear regression program does not provide initial estimates of the variables, enter these values. Span is the specific binding at time zero and is estimated as the first $y$ value minus the last $y$ value. Plateau is the binding after a long time, and reflects nonspecific binding, which does not change with time. Estimate it as the last $y$ value. $\mathrm{K}$ is the dissociation rate constant $\left(k_{\text {off }}\right)$. Estimate it by dividing 0.69 by an estimate of the half-time of dissociation.

5. Start the nonlinear regression procedure.

6. Calculate the half-life of dissociation from the rate constant.

$$
\text { half-life }=\ln (2) / k_{\text {off }}=0.693 / \mathrm{K}
$$

\section{Using nonlinear regression to determine $\boldsymbol{k}_{\text {on }}$}

1 . Enter the $x$ data as time in minutes.

2. Enter the $y$ data as specific binding in cpm.

3. Choose the exponential association equation. It may be expressed as:

$$
y=y_{\max } \times\{1-\exp (-\mathrm{K} \times x)\}
$$

4. If the nonlinear regression program does not provide initial estimates of the variables, enter these values. $y_{\max }$ is the specific binding at equilibrium, which is estimated as the last $y$ value. $\mathrm{K}$ is the observed rate of association $\left(k_{\mathrm{ob}}\right)$. Estimate it by dividing 0.69 by an estimate of the time it takes to achieve half-maximal binding.

5. Start the nonlinear regression procedure to determine $k$ and $y_{\max }$. Note that $k$ is the observed rate constant $\left(k_{\mathrm{ob}}\right)$ in units of inverse time $\left(\mathrm{min}^{-1}\right)$ if you entered $x$ in minutes. This is not the same as the association rate constant.

6. Calculate the association rate constant from the observed rate constant and the dissociation rate constant. Use the following equation, where [radioligand] is expressed in molar and $k_{\mathrm{ob}}$ and $k_{\mathrm{off}}$ are expressed in $\mathrm{min}^{-1}$.

$$
k_{\mathrm{on}}=\left(k_{\mathrm{ob}}-k_{\mathrm{off}}\right) /[\text { radioligand }]
$$




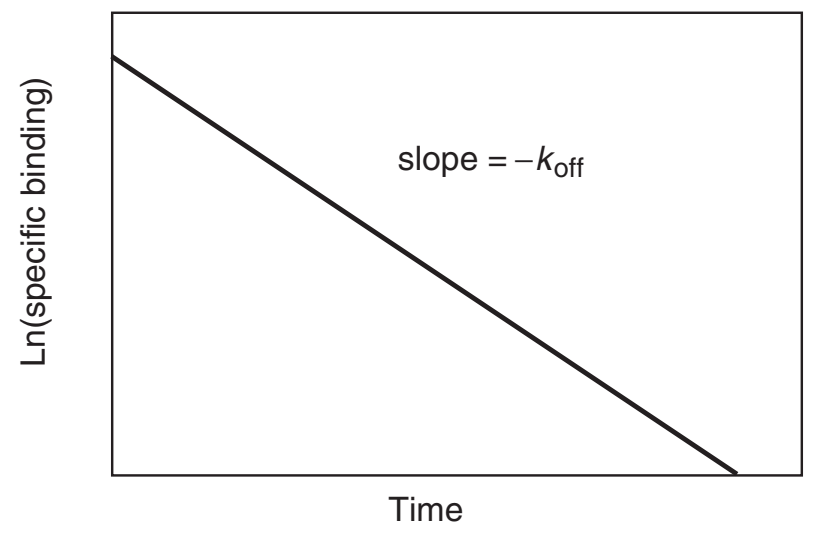

Figure 7.5.16 Schematic of a dissociation kinetic experiment shown on a log scale. The $y$ axis plots the natural log of specific binding.

\section{Displaying dissociation data on a log plot}

Figure 7.5.16 shows a plot of $\ln \left(B_{t} / B_{0}\right)$ versus time. The graph of a dissociation experiment will be linear if the system follows the law of mass action with a single affinity state. $B_{t}$ is the specific binding at time $t ; B_{0}$ is specific binding at time zero. The slope of this line will equal $-k_{\text {off }}$.

The log plot will only be linear when taking the logarithm of specific binding as a fraction of binding at time zero. Don't use total binding.

Use the natural logarithm, not the base ten log in order for the slope to equal $-k_{\mathrm{off}}$. If you use the base $10 \log$, then the slope will equal -2.303 times $k_{\text {off }}$.

Use the log plot only to display data, not to analyze data. A more accurate rate constant will be obtained by fitting the raw data using nonlinear regression.

\section{Interpreting the Results}

\section{Are the results reasonable?}

Table 7.5.6 presents some questions that should be addressed when determining if the results are reasonable.

\section{Using Kinetic Data to Test the Law of Mass Action}

Standard binding experiments are usually fit to equations derived from the law of mass action. Kinetic experiments provide a more sensitive test than equilibrium experiments to determine whether the law of mass action actually applies for the system of interest. To test the law of mass action, ask these questions:

Does the $K_{d}$ calculated from kinetic data match the $K_{d}$ calculated from saturation binding?

According to the law of mass action, the ratio of $k_{\text {off }}$ to $k_{\text {on }}$ is the $K_{\mathrm{d}}$ of receptor binding:

$$
K_{\mathrm{d}}=\frac{k_{\text {off }}}{k_{\text {on }}}
$$

The units are consistent: $k_{\text {off }}$ is in units of $\min ^{-1}$ and $k_{\text {on }}$ is in units of $\mathrm{M}^{-1} \mathrm{~min}^{-1}$, so $K_{\mathrm{d}}$ is in units of $\mathrm{M}$.

Neurochemistry/ Neuropharmacology

7.5.27 
If binding follows the law of mass action, the $K_{\mathrm{d}}$ calculated in this way should be the same as the $K_{\mathrm{d}}$ calculated from a saturation binding curve.

\section{Does $\boldsymbol{k}_{o b}$ increase linearly with the concentration of radioligand?}

The observed association rate constant, $k_{\mathrm{ob}}$, is defined by this equation:

$$
k_{\mathrm{ob}}=k_{\mathrm{off}}+k_{\mathrm{on}} \times[\text { radioligand }]
$$

Therefore, association rate experiments performed at various concentrations of radioligand should look like Figure 7.5.17. As the concentration of radioligand is increased, the observed rate constant increases linearly. If the binding is more complex than a simple mass action model (such as a binding step followed by a conformational change) the plot of $k_{\mathrm{ob}}$ vs. [radioligand] may plateau at higher radioligand concentrations. The $y$ intercept of the line equals $k_{\text {off }}$. If the law of mass action applies to the system, the $k_{\text {off }}$ determined in this way should correspond to the $k_{\text {off }}$ determined from a dissociation experiment. Finally, this kind of experiment provides a more rigorous determination of $k_{\mathrm{on}}$ than the value obtained with a single concentration of radioligand.

\section{Is specific binding $100 \%$ reversible, and is the dissociated ligand chemically intact?}

Nonspecific binding at "time zero" should equal total binding at the end (plateau) of the dissociation. In other words, all of the specific binding should dissociate after a sufficiently long period of time. Use chromatography to analyze the radioligand that dissociates to prove that it has not been altered.

Table 7.5.6 Evaluating the Results of Association Binding Analyses

\begin{tabular}{ll}
\hline Question & Comment \\
\hline $\begin{array}{l}\text { Was data collected over a long enough } \\
\text { period of time? }\end{array}$ & $\begin{array}{l}\text { Dissociation and association data should plateau, so } \\
\text { the data obtained at the last few time points should } \\
\text { be indistinguishable. }\end{array}$ \\
Is the value of $k_{\text {on }}$ reasonable? & $\begin{array}{l}\text { The association rate constant, } k_{\text {on }} \text {, depends largely } \\
\text { on diffusion, so the value is similar for many } \\
\text { ligands. Expect a result of } \sim 10^{8} \mathrm{M}^{-1} \mathrm{~min}^{-1} .\end{array}$
\end{tabular}

Is the value of $k_{\text {off }}$ reasonable? If the $k_{\text {off }}$ is $>1 \mathrm{~min}^{-1}$, the ligand has a low affinity for the receptor. Most likely, dissociation will occur during separation of bound and free ligands. If $k_{\text {off }}$ is $<0.001 \mathrm{~min}^{-1}$, attaining equilibrium will be difficult as the half-time of dissociation will be greater than $10 \mathrm{hr}$ ! Even if one waits that long, other reactions may occur that ruin the experiment.

Are the standard errors too large? Are the confidence intervals too wide?

Examine the SE and the confidence intervals to gauge the level of confidence to give the rate constants.

Does only a tiny fraction of radioligand bind to the receptors?

The standard analyses of association experiments assume that the concentration of free radioligand is constant during the experiment. This will be approximately true only if a tiny fraction of the added radioligand binds to the receptors. Compare the maximum total binding in $\mathrm{cpm}$ to the amount of added radioligand in cpm. If that ratio exceeds $\sim 10 \%$, revise the experimental protocol. 


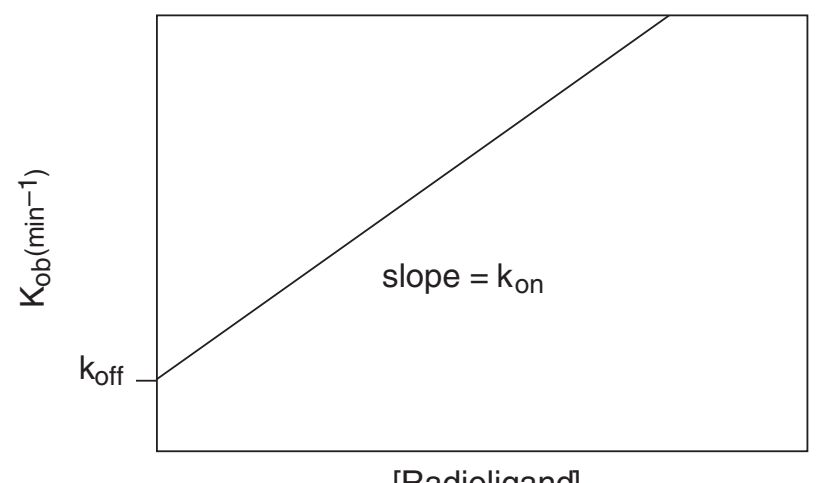

[Radioligand]

Figure 7.5.17 Schematic of observed association rate constants as a function of radioligand concentration. Higher concentrations of radioligand equilibrate more quickly. The slope of the line equals the association rate constant $\left(k_{\mathrm{on}}\right)$; the $y$ intercept is the dissociation rate constant $\left(k_{\mathrm{off}}\right)$.

\section{Is the dissociation rate the same when dissociation is initiated from different amounts or times of receptor occupation?}

If the ligand binds to a single site and obeys the law of mass action, the dissociation rate constant is independent of the amount of radioligand used or the time before initiating dissociation.

\section{Is there cooperativity?}

If the law of mass action applies, binding of a ligand to one binding site does not alter the affinity of another binding site. This also means that dissociation of a ligand from one site should not change the dissociation of ligand from other sites. To test this assumption, compare dissociation initiated by adding an unlabeled ligand with dissociation initiated by infinite dilution. The two rate constants should be identical (see Competitive Binding with Two Sites, below).

\section{Kinetics of Competitive Binding}

The standard methods of kinetic binding determine the $k_{\text {on }}$ and $k_{\text {off }}$ for a labeled ligand. Competitive binding can be used to determine the $k_{\text {on }}$ and $k_{\text {off }}$ of an unlabeled ligand. Add the two ligands at the same time, and measure radioligand binding over time. Use the following information to set up the equation (Motulsky and Mahan, 1984).

Define the following variables.

$\boldsymbol{k 1}$ association rate constant of radioligand $\left(\mathrm{M}^{-1} \mathrm{~min}^{-1}\right)$

$\boldsymbol{k 2}$ dissociation rate constant of radioligand $\left(\mathrm{min}^{-1}\right)$

$\boldsymbol{k 3}$ association rate constant of unlabeled ligand $\left(\mathrm{M}^{-1} \min ^{-1}\right)$

$\boldsymbol{k} \mathbf{4}$ dissociation rate constant of unlabeled ligand $\left(\mathrm{min}^{-1}\right)$

[radioligand] concentration of labeled drug (M)

[unlabeled drug] concentration of unlabeled drug (M)

$S$ an arbitrary designation of an intermediate variable

$\boldsymbol{B}_{\text {max }}$ total number of binding sites (same units as specific binding, usually cpm)

Neurochemistry/ Neuropharmacology

7.5.29 


$$
\begin{aligned}
& \boldsymbol{t} \text { time (min) } \\
& K_{\mathrm{A}}=k_{1} \times[\text { radioligand }]+k_{2} \\
& K_{\mathrm{B}}=k_{3} \times[\text { unlabeled ligand }]+k_{4} \\
& \mathrm{~S}=\sqrt{\left(K_{\mathrm{A}}-K_{\mathrm{B}}\right)^{2}+4 \times k_{1} \times k_{3} \times[\text { radioligand }] \times[\text { unlabeled ligand }]} \\
& K_{\mathrm{F}}=\frac{K_{\mathrm{A}}+K_{\mathrm{B}}+\mathrm{S}}{2} \quad K_{\mathrm{S}}=\frac{K_{\mathrm{A}}+K_{\mathrm{B}}-\mathrm{S}}{2} \\
& \text { specific binding }= \\
& \quad \frac{B_{\mathrm{max}} \times k_{1} \times[\text { radioligand }]}{K_{\mathrm{F}}-K_{\mathrm{S}}}\left[\frac{k_{4} \times\left(K_{\mathrm{F}}-K_{\mathrm{S}}\right)}{K_{\mathrm{F}} \times K_{\mathrm{S}}}+\frac{k_{4}-K_{\mathrm{F}}}{K_{\mathrm{F}}} \times e^{-K_{\mathrm{F}} \cdot t}-\frac{k_{4}-K_{\mathrm{S}}}{K_{\mathrm{S}}} \times e^{-K_{\mathrm{S}} \cdot t}\right]
\end{aligned}
$$

Many data points are needed at early time points for this method to work. When fitting the data, set $k_{1}$ and $k_{2}$ to constant values determined from standard kinetic experiments. Set $B_{\max }$ to a constant value determined in a saturation binding experiment. The concentrations of labeled and unlabeled compound are also constants, set by your experimental design. Fit the data to determine $k_{3}$ and $k_{4}$.

\section{TWO BINDING SITES}

Several receptor molecules frequently evolve for a single hormone or neurotransmitter. Also, many ligands bind to more than one receptor subtype.

\section{Saturation Binding Experiments with Two Sites}

When the radioligand binds to two classes of receptors, analyze the data by using this equation.

$$
\text { specific binding }=y=\frac{B_{\max 1} \times[\mathrm{L}]}{K_{\mathrm{d} 1}+[\mathrm{L}]}+\frac{B_{\max 2} \times[\mathrm{L}]}{K_{\mathrm{d} 2}+[\mathrm{L}]}
$$

Panel A of Figure 7.5.18 shows specific binding to two classes of receptors present in equal quantities, whose $K_{\mathrm{d}}$ values differ by a factor of ten. Panel B shows the transformation to a Scatchard plot. In both graphs the dotted and dashed lines show binding to the two individual receptors; the sum in each graph is represented by a solid curve.

Note that the graph of specific binding is not obviously biphasic. It is very hard to see the presence of two binding affinities by just looking. The best way to detect the second site is to fit data to one- and two-site curves, and let the nonlinear regression program compare the two fits (see Theory: Comparing One- and Two-Site Models, below). The curvature of the Scatchard plot is not dramatic and can easily be obscured by experimental scatter. Note the location of the solid and dashed line in the Scatchard plot. The two components of a biphasic Scatchard are not the asymptotes of the curve.

\section{Competitive Binding with Two Sites}

Competitive binding experiments are often used in systems where the tissue contains two classes of binding sites (e.g., two subtypes of a receptor). Analysis of these data are straightforward if the following assumptions are met:

Analyzing Radioligand Binding Data
1. There are two distinct classes of receptors. For example, a tissue could contain a mixture of $\beta_{1}$ and $\beta_{2}$ adrenergic receptors. 
2. The unlabeled ligand has distinct affinities for the two sites.

3. The labeled ligand has equal affinity for both sites.

4. Binding has reached equilibrium.

5. A small fraction of both labeled and unlabeled ligand bind. This means that the concentration of labeled and unlabeled ligand added is very close to the free concentration in all tubes.

Based on these assumptions, binding follows the equation:

$$
y=\mathrm{NS}+(\text { Total }-\mathrm{NS})\left[\frac{\mathrm{F}}{1+10^{\log [\mathrm{D}]-\log \left(\mathrm{IC}_{50} \mathrm{~A}\right)}}+\frac{1-\mathrm{F}}{1+10^{\log [\mathrm{D}]-\log \left(\mathrm{IC}_{50} \mathrm{~B}\right)}}\right]
$$

This equation has five variables: the total and nonspecific binding (the top and bottom binding plateaus), the fraction of binding to receptors of the first type of receptor $(F)$, and the $\mathrm{IC}_{50}$ of the unlabeled ligand for each type of receptor. If the $K_{\mathrm{d}}$ and concentration of

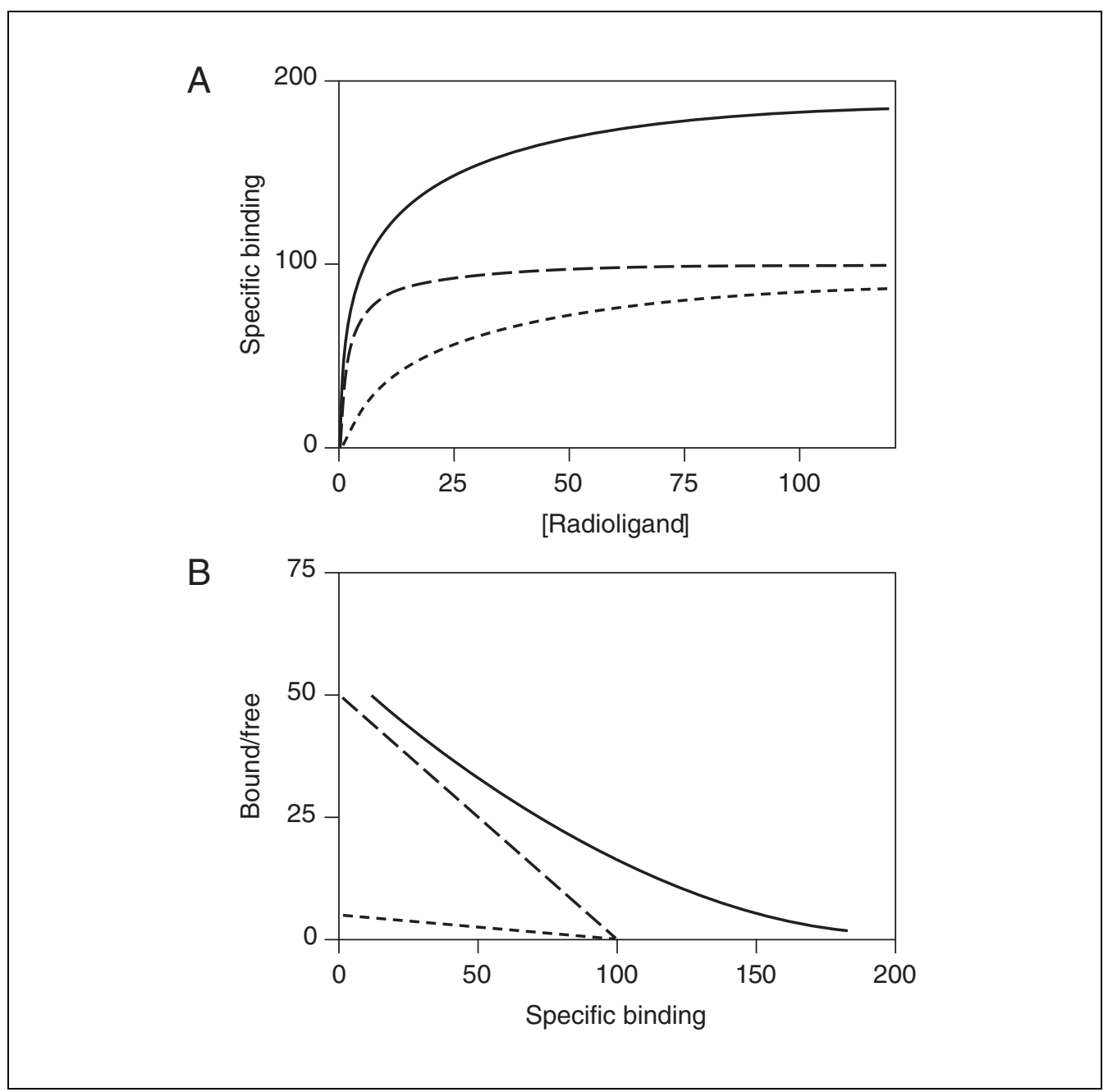

Figure 7.5.18 Saturation binding to two classes of receptors. The two receptor types are present in equal quantities, but have $K_{d}$ values that differ by a factor of ten. (A) Binding to the two individual receptor types are shown as dashed curves. The sum (observed experimentally) is shown as a solid curve. It is not obviously biphasic. (B) Scatchard transformation. The curvature of the overall Scatchard plot (solid) is subtle, and it would be easy to miss the curvature if the data were scattered. Note that the Scatchard plots for the individual receptors (dashed) are notasymptotes of the two-site Scatchard plot (solid).

Neurochemistry/ Neuropharmacology 
the labeled ligand is known, the $\mathrm{IC}_{50}$ values can be converted to $K_{\mathrm{i}}$ values (see Analyzing Competitive Binding Data, above).

Since there are two different kinds of receptors with different affinities, a biphasic competitive binding curve might be expected. In fact, a biphasic curve is seen only in unusual cases where the affinities are extremely different. More often, the two components are blurred together into a shallow curve. For example, Figure 7.5.19 shows competition for two equally abundant sites with a tenfold (one log unit) difference in $\mathrm{IC}_{50}$. Careful observation will reveal that the curve is shallow (it takes more than two log units to go from $90 \%$ to $10 \%$ competition), but two distinct components are not visible.

\section{Cooperativity}

In the standard mass action model, each binding site is independent. The standard mass action assumes that there is no cooperativity. Cooperativity occurs when binding of a ligand to one binding site affects binding to adjacent sites. Usually these binding sites are on the same molecule. If binding of one ligand increases the affinity of an adjacent site, this is positive cooperativity. If binding of one ligand decreases the affinity of an adjacent site, this is negative cooperativity. It is impossible to distinguish negative cooperativity from multiple independent binding sites (with different affinities) from data collected at equilibrium. Kinetic experiments are needed.

To distinguish between multiple independent binding sites and negative cooperativity, compare the dissociation rate after initiating dissociation by infinite dilution with the dissociation rate when initiated by addition of a large concentration of unlabeled drug. If the radioligand is bound to multiple noninteracting binding sites, the dissociation will be identical in both experimental protocols as shown in panel A of Figure 7.5.20. Note that the $y$ axis is shown using a log scale. If there were a single binding site, the dissociation data would be expected to appear linear on this graph. With two binding sites, the graph is curved, even on a log axis (assuming the radioligand is present at high enough concentration to bind appreciably to both sites).

Panel B shows ideal dissociation data when radioligand is bound to interacting binding sites with negative cooperativity. The data are different depending on how dissociation was initiated. If dissociation is initiated by infinite dilution, the dissociation rate will change over time. The dissociation of some radioligand will leave the remaining ligand bound more tightly. When dissociation is initiated by addition of cold drug, all the

Analyzing Radioligand Binding Data

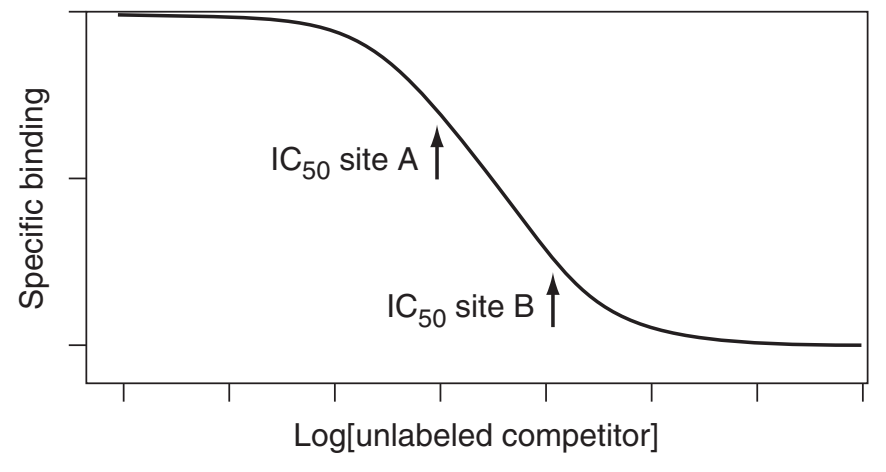

Figure 7.5.19 Two site competitive binding curve. The radioligand binds identically to two kinds of receptors, but these two receptors have a tenfold difference in affinity for the competitor. The curve is shallow, but not obviously biphasic. 
receptors are always occupied by ligand (some hot, some cold) and dissociation occurs at its maximal unchanging rate.

\section{Theory: Comparing One- and Two-Site Models}

\section{Why not just compare sum of squares or $R^{2}$ ?}

In a least squares analysis of data (either linear or nonlinear), the computer program will give an $R^{2}$ value and the sum of the squared deviations from the theoretical fit in the experimental result. The smaller the sum-of-squares value and the higher the $R^{2}$, the better the theory fits the data. However, a two-site model will almost always fit the data better than a one-site model. A three-site model fits even better, and a four-site model better yet! As more variables (sites) are added to the equation, more inflection points are added to the curve, so it gets closer to the points. The sum of squares gets smaller; $R^{2}$ gets higher. Statistical calculations (such as the $F$ test described below) should be used to see whether these changes are larger than expected by chance.

\section{Reality check}

Before performing statistical comparisons, however, look at whether the results make sense. Sometimes the two-site fit gives results that are clearly nonsense. Disregard a two-site fit when: (1) the two $\mathrm{IC}_{50}$ or $K_{\mathrm{d}}$ values are almost identical — the data are probably fit quite well by a single-site model; (2) one of the $\mathrm{IC}_{50}$ or $K_{\mathrm{d}}$ values is outside the range of data; (3) one of the sites has a very small fraction of the receptors-if there are too few sites, the $\mathrm{IC}_{50}$ or $K_{\mathrm{d}}$ cannot be determined reliably. (4) The best-fit values for the bottom and top plateaus are far from the range of $y$ values observed in the experiment (applies to competitive binding curves only).

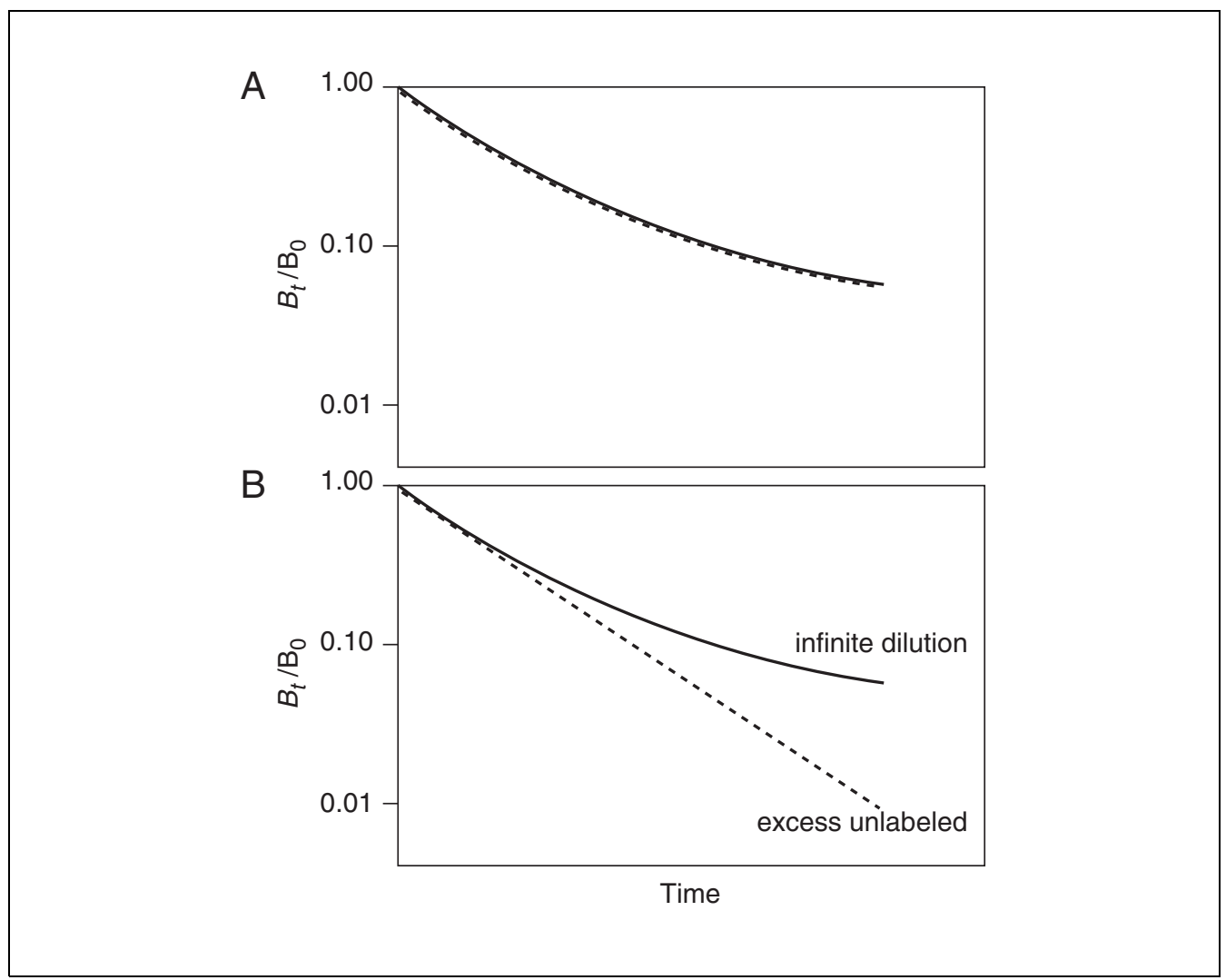

Figure 7.5.20 Discriminating between binding to two (or more) binding sites (top) and negative cooperativity. With negative cooperativity, dissociation will be faster when initiated by adding excess unlabeled ligand than when initiated by infinite dilution.

Neurochemistry/ Neuropharmacology

7.5.33 
If the two-site fit seems reasonable, test whether the difference between the one- and two-site fit is statistically significant.

\section{Using the F test to compare one- and two-site fits}

Even if the simpler one-site model is correct, the fit is expected to be worse (have the higher sum of squares) because it has fewer inflection points (more degrees of freedom). In fact, statisticians have proven that the relative increase in the sum of squares (SS) is expected to equal the relative increase in degrees of freedom (DF). In other words, if the one-site model is correct it would be expected that:

$$
(\mathrm{SS} 1-\mathrm{SS} 2) / \mathrm{SS} 2 \approx(\mathrm{DF} 1-\mathrm{DF} 2) / \mathrm{DF} 2
$$

If the more complicated two-site model is correct, then the relative increase in sum of squares (going from two-site to one-site) is expected to be greater than the relative increase in degrees of freedom:

$$
(\mathrm{SS} 1-\mathrm{SS} 2) / \mathrm{SS} 2>(\mathrm{DF} 1-\mathrm{DF} 2) / \mathrm{DF} 2
$$

Follow these steps to compare the two models:

1. Fit the data to the simpler (one-site) model and record the sum of squares (SS1) and degrees of freedom (DF1).

2. Fit the data to the more complicated (two-site) model and record the sum of squares (SS2) and degrees of freedom (DF2).

3. Look at whether the two-site model makes sense. If the best-fit values don't make sense (or the values for the two sites are almost the same), then discard the two-site model and accept the one-site model.

4. Compare SS2 with SS1. If for some reason SS2 is larger than SS1, then the two-site fit is worse than the one-site fit and should be discarded. Accept the one-site fit. In most cases SS1 is larger, and further calculations will be needed.

5. Calculate the $F$ ratio, which quantifies the relationship between the relative increase in sum of squares and the relative increase in degrees of freedom.

$$
F=\frac{(\mathrm{SS} 1-\mathrm{SS} 2) / \mathrm{SS} 2}{(\mathrm{DF} 1-\mathrm{DF} 2) / \mathrm{DF} 2}
$$

The equation for calculating $F$ is usually presented in this equivalent form (see Table 7.5.7 for corresponding ANOVA table).

$$
F=\frac{(\mathrm{SS} 1-\mathrm{SS} 2) /(\mathrm{DF} 1-\mathrm{DF} 2)}{\mathrm{SS} 2 / \mathrm{DF} 2} \quad \mathrm{DF}_{\mathrm{n}}=(\mathrm{DF} 1-\mathrm{DF} 2), \quad \mathrm{DF}_{\mathrm{d}}=\mathrm{DF} 2
$$

Table 7.5.7 ANOVA Table for Comparison of One- and Two-Site Fits ${ }^{a}$

Analyzing Radioligand Binding Data

\begin{tabular}{llll}
\hline Source of variation & Sum of squares & DF & $\begin{array}{l}\text { Mean } \\
\text { square }\end{array}$ \\
\hline Difference & SS1 - SS2 & DF1 - DF2 & SSI - SS2 \\
\cline { 2 - 3 } Model 2 (complicated) & SS2 & DF1 - DF2 & SS2/DF2 \\
Model 1 (simple) & SS1 & DF1 & \\
\hline
\end{tabular}

${ }^{a}$ ANOVA, analysis of variance. 


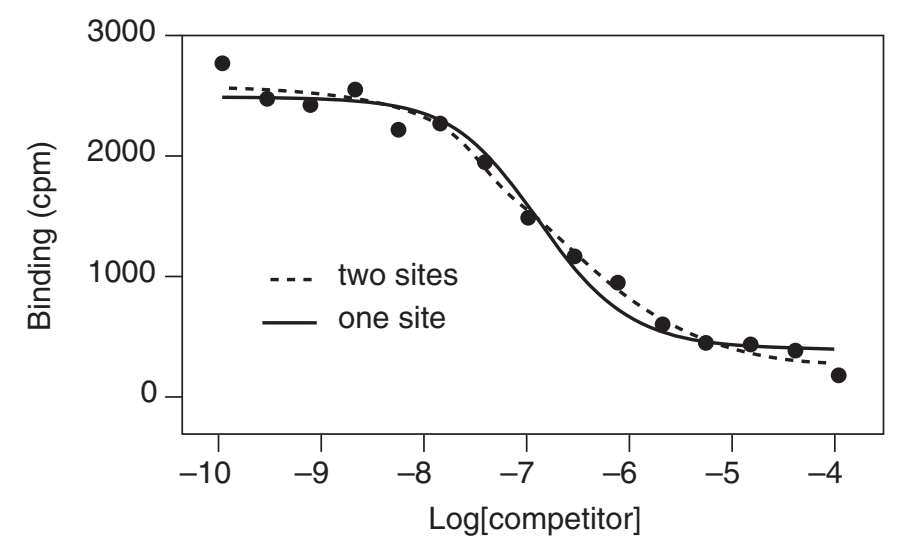

Figure 7.5.21 The solid curve shows the fit to an equation describing competition for a single class of receptors. The dashed curve shows the fit to an equation describing competition for binding to two classes of receptors.

6. Use a table or program to determine the $P$ value. When doing so, degrees of freedom should be entered for both the numerator $\left(\mathrm{DF}_{\mathrm{n}}\right)$ and denominator $\left(\mathrm{DF}_{\mathrm{d}}\right)$. The numerator has (DF1 - DF2) degrees of freedom. The denominator has DF2 degrees of freedom.

If the one-site model is correct, an $F$ ratio near one and a large $P$ value are expected. If the two-site fit is correct, a large $F$ ratio and a small $P$ value would be seen. The $P$ value can be small for two reasons. One possibility is that the two-site model is correct. The other possibility is that the one-site model is correct, but random scatter led the two-site model to fit better by chance. The $P$ value tells how rarely this coincidence would occur. More precisely, the $P$ value answers this question: If the one-site model is really correct, what is the chance that data would randomly fit the two-site model so much better?

If the $P$ value is smaller than a preset threshold (set to the arbitrary value of 0.05 by tradition), conclude that the two-site model is significantly better than the one-site model.

Figure 7.5.21 compares a one-site and two-site competitive binding curve. The results are shown in Table 7.5.8.

Table 7.5.8 Comparison of One-Site and Two-Site Competitive Binding Curve

\begin{tabular}{lccc}
\hline & Two-site & One-site & $\%$ increase \\
\hline Degrees of freedom & 10 & 12 & 20.00 \\
Sum of squares & 129,800 & 248,100 & 91.14 \\
\hline
\end{tabular}

In going from the two-site to the one-site model, two degrees of freedom are gained, because the one-site model has two fewer variables. Since the two-site model has 10 degrees of freedom ( 15 data points minus 5 variables), the degrees of freedom increased $20 \%$. If the one-site model were correct, the sum of squares would be expected to increase $\sim 20 \%$ just by chance. In fact, the sum of squares increased $91 \%$. The percent increase was 4.56 times higher than expected $(91.1 / 20.0=4.56)$. This is the $F$ ratio $(F=4.56)$, and it corresponds to a $P$ value of 0.039 . If the one-site model is correct, there is only a $3.9 \%$

Neurochemistry/ Neuropharmacology

7.5.35 


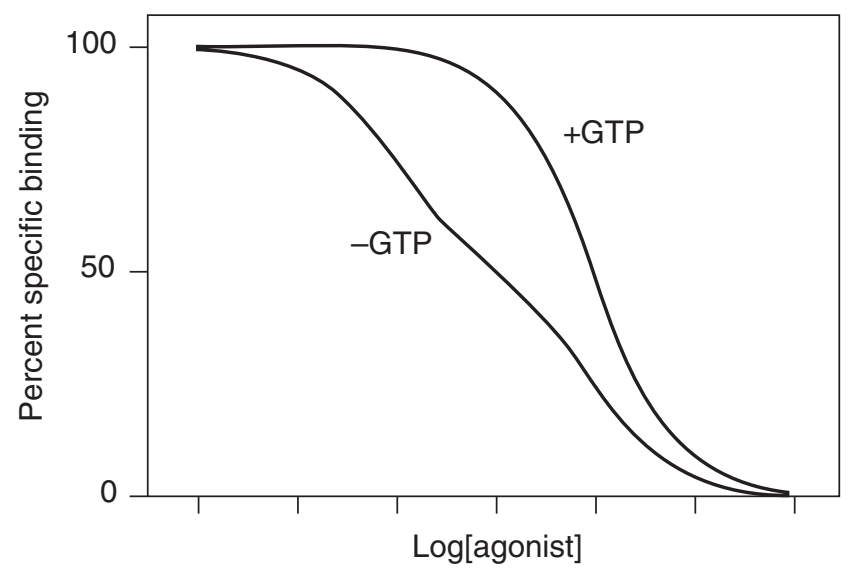

Figure 7.5.22 Schematic of agonist competition for binding to a receptor linked to a G protein. In the absence of GTP (left) the curve is shallow (and in this extreme case, biphasic). In the presence of GTP (or an analog) the curve is shifted to the right and is steeper.

chance that randomly obtained data would fit the two-site model so much better. Since this is below the traditional threshold of 5\%, conclude that the two-site model fits significantly better than the one-site model.

\section{AGONIST BINDING}

\section{Receptors Linked to G Proteins}

The most studied example of agonist binding is the interaction of agonists with receptors that are linked to $\mathrm{G}$ proteins. This is studied by comparing the competition of agonists with radiolabeled antagonist binding in the presence and absence of GTP (or its analogs). These experiments are done in membrane preparations to wash away endogenous intracellular GTP. Without added GTP, the competitive binding curves tend to be shallow. When GTP or an analog is added, the competitive binding curve is of normal steepness. Figure 7.5.22 shows the results of an idealized experiment.

The extended ternary complex model can partially account for these findings (and others). In this model, receptors can exist in two states, $\mathrm{R}$ and $\mathrm{R} *$. The $\mathrm{R}^{*}$ state has a high affinity for agonist and preferentially associates with $G$ proteins to form an $R * G$ complex. Although some receptors may exist in the $\mathrm{R}^{*}$ state in the absence of agonist, the binding of agonist fosters the transition from $\mathrm{R}$ to $\mathrm{R}^{*}$, and thus promotes interaction of the receptor with $G$ protein to form the ternary complex $\mathrm{HR}^{*} \mathrm{G}$. The extended ternary complex model is shown in Figure 7.5.23.

The agonist binding curve is shallow (showing high and low affinity components) in the absence of GTP because some receptors interact with $\mathrm{G}$ proteins and others do not. The receptors that do interact with $G$ proteins bind agonist with high affinity, while those that do not interact bind with low affinity. Not all receptors can bind to $G$ proteins because either the receptors are heterogeneous, the $G$ proteins are limiting, or the membrane compartmentation prevents some receptors from interacting with $G$ proteins. If all the receptors could interact with $G$ proteins, the expectation would be a high affinity, competitive binding curve in the absence of GTP. In the presence of GTP (or an analog),

Analyzing Radioligand Binding Data the $\mathrm{HR}^{*} \mathrm{G}$ complex is not stable, so the $\mathrm{G}$ protein dissociates into its $\alpha_{\mathrm{GTP}}$ and $\beta \gamma$ subunits, and is uncoupled from the receptor. With GTP present, only a tiny fraction of receptors 


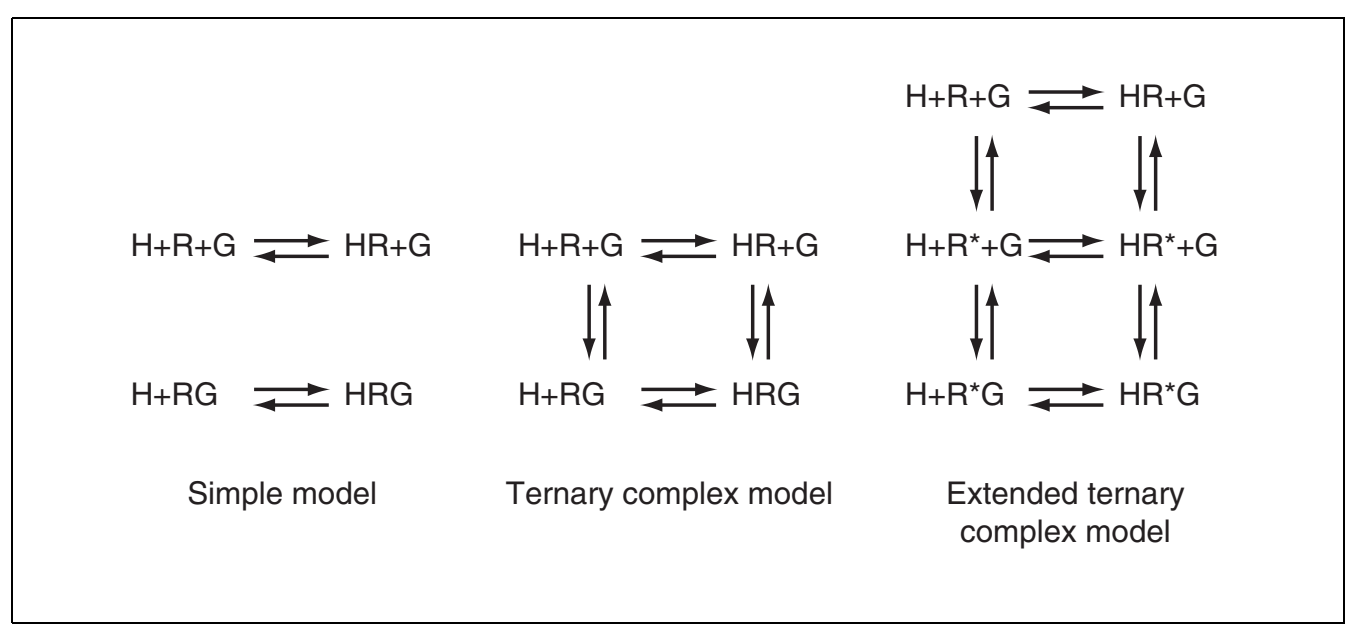

Figure 7.5.23 Models for agonist binding to receptors linked to $G$ proteins. $H$, hormone or agonist; $\mathrm{R}$, receptor; G, G protein.

are coupled to $\mathrm{G}$ at any given time, so the agonist competition curves are of low affinity and normal steepness as if only $\mathrm{R}$ was present and not RG.

Although the extended ternary complex model is very useful conceptually, it is not very useful when analyzing data. There are simply too many variables. The simpler ternary complex model shown in Figure 7.5.23 has fewer variables, but still too many to reliably fit with nonlinear regression. For routine analyses, most investigators fit data to the much simpler two-state model shown in the figure. This model allows for receptors to exist in two affinity states ( $R$ and $R G$ ), but does not allow conversion between them. It is easy to fit data to this simpler model using a two-site competition curve model. Since the model is too simple, the high and low affinity dissociation constants derived from the model should be treated merely as empirical descriptions of the data and should not be thought of as true molecular equilibrium constants.

\section{Other Kinds of Receptors}

By definition, the binding of agonists to receptors makes something happen. So it is not surprising that agonist binding is often more complicated than the simple mass action model. For example, binding of agonists to nicotinic acetylcholine receptor causes a conformational change characterized by a high affinity binding of the agonist and desensitized receptors, and insulin binding to its receptor shows negative cooperativity due to dimerization of the receptors.

\section{ANALYZING DATA USING NONLINEAR REGRESSION}

Radioligand binding data are best analyzed using nonlinear regression to fit curves through the data.

\section{The Problem with Using Linear Regression on Transformed Data}

Before the age of microcomputers, scientists transformed their data to make a linear graph, and then analyzed the transformed data with linear regression. Examples include Lineweaver-Burke plots of enzyme kinetic data, Scatchard plots of binding data, and logarithmic plots of kinetic data.

These methods are outdated, and should not be used to analyze data. The problem is that the linear transformation distorts the experimental error. Linear regression assumes that the scatter of points around the line follows a Gaussian distribution and that the standard

Neurochemistry/ Neuropharmacology

7.5.37 
deviation is the same at every value of $x$. These assumptions are usually not true with transformed data. A second problem is that some transformations alter the relationship between $x$ and $y$. For example, in a Scatchard plot the value of $x$ (bound) is used to calculate $y$ (bound/free), and this violates the assumptions of linear regression. For an example of this, see Analysis of Saturation Binding Curves, The Problem with Using Scatchard Plots.

Since the assumptions of linear regression are violated, the results of linear regression are incorrect. The values derived from the slope and intercept of the regression line are not the most accurate determinations of the receptor number, rate constants, or dissociation constants. Considering all the time and effort put into collecting data, the best possible analysis technique should be used, and nonlinear regression produces the most accurate results.

Although linear regression is usually inappropriate for analyzing transformed data, it is often helpful for displaying transformed data because many people find it easier to visually interpret linear data. This makes sense because the human eye and brain evolved to detect edges (lines), not to detect rectangular hyperbolas or exponential decay curves.

\section{Comparison of Linear and Nonlinear Regression}

A line is described by a simple equation that calculates $y$ from $x$, slope, and intercept. The purpose of linear regression is to find values for the slope and intercept that define the line that best fits the data. More precisely, it finds the line that minimizes the sum of the squares of the vertical distances of the points from the line.

The goal of minimizing the sum of squares in linear regression can be achieved quite simply. A bit of algebra (shown in many statistics books) derives equations that define the best-fit slope and intercept. Put the data in, do a few calculations, and the answers come out. There is no chance for ambiguity.

Nonlinear regression fits data to any equation that defines $y$ as a function of $x$ and one or more variables. Like linear regression, it finds the values of those variables that minimize the sum of the squares of the vertical distances of the points from the curve. With the exception of a few special cases (like linear regression), it is not possible to solve the equations directly to find the best-fit values of the variables. Instead nonlinear regression requires an iterative approach that requires use of a computer.

To analyze data with nonlinear regression, the program will require an equation (model) that defines $y$ as a function of $x$ and one or more variables (i.e., $K_{\mathrm{d}}, B_{\max }$, or rate constants). It will also require an estimate (or guess) for the best-fit value for each variable in the equation (some programs provide the initial estimates automatically).

Every nonlinear regression program follows these steps:

1. Using the initial values provided, the program calculates a predicted value of $y$ for each value of $x$. It then compares the actual $y$ values with the predicted $y$ values, and calculates the sum of the squares of the differences between observed and predicted $y$ values.

2. The program then adjusts the variables to improve the fit and reduce the sum of squares. There are several algorithms for adjusting the variables. The most commonly used method was derived by Levenberg and Marquardt (often called simply the Marquardt method), but the details of how this method works cannot be understood without first mastering matrix algebra. However, nonlinear regression can be used to analyze data without knowing anything about these algorithms. 
3. Step 2 is repeated several times. Each time the variables are adjusted by a smaller amount. Stop when the adjustments make virtually no difference in the sum of squares. This typically requires 5 to 20 iterations.

4. Best-fit results are reported. The precise values obtained will depend in part on the initial values and the stopping criteria. This means that repeat analyses of the same data will not always give exactly the same results.

\section{Picking a Nonlinear Regression Program}

When choosing a program to analyze binding data, beware of these three traps:

1. Rather than requesting an equation, some programs automatically fit data to hundreds or thousands of equations and then present the equation(s) that fit the data best. Using such a program is appealing because it frees the user from the need to choose an equation. The problem is that the program has no scientific understanding of the experiment and uses equations that do not correspond to any model of binding. It will not be possible to interpret the best-fit values of the variables in terms of rate constants or affinities.

2. Many so-called curve fitting programs actually fit data to a polynomial equation: $y$ $=\mathrm{A}+\mathrm{B} x+\mathrm{C} x^{2}+\mathrm{D} x^{3} \ldots$. Since the binding of ligands to receptors cannot be expressed as a polynomial equation, polynomial regression is not useful for analyzing binding data.

3. Some programs fit curves by drawing a cubic spline curve - a smooth curve that goes through every data point. In some cases, a cubic spline curve can look attractive on a graph and it may work well as a standard curve for interpolation. However, the curve does not correspond to any model, so cubic spline is not useful in data analysis.

Once the search is narrowed to programs that perform nonlinear regression to fit data to a selected equation, ask the following questions:

1. Can common binding equations be selected from a menu, or must the equations be entered into a file?

2. Does the program provide initial values automatically (see below), or will they have to be entered for each analysis?

3. Can the program automatically compare two models with the $F$ test?

4. Does the program prepare publication-quality graphs of the data and curves?

5. When the data are saved to file, are the analysis choices and results saved as well? This is important to retrace the analysis steps in the future.

6. Is the program designed specifically for analyses of binding data? The program Ligand (Munson and Rodbard, 1980) was designed just for analyses of binding studies, and can handle situations that many other programs cannot (i.e., simultaneous analyses of several experiments; correcting for ligand depletion), although its interface is no longer state-of-the-art.

One nonlinear regression program, GraphPad Prism (designed by the authors of this chapter), is particularly well suited for analyses of radioligand binding data (see Analyzing Data with GraphPad Prism, below). 


\section{Decisions That Need to be Made When Fitting Curves with Nonlinear Regression}

When using a program for nonlinear regression, the following decisions must be made.

\section{Which equation?}

An equation must be chosen that defines $y$ as a function of $x$ and one or more variables. This equation should represent a model, usually the law of mass action. Later sections of this unit show equations that describe the law of mass action in various kinds of binding experiments.

\section{Which units?}

In pure mathematics, it doesn't matter whether data is entered as 1 picomolar or $10^{-12}$ molar, as $100 \mathrm{fmol} / \mathrm{mg}$ or $60,000,000,000$ receptors $/ \mathrm{mg}$. When computers do the calculating, however, it can matter. Calculation problems such as round off errors are far more likely when the values are very high or very low. Scale data to avoid values $<10^{-4}$ or $>10^{4}$.

\section{Estimate initial values}

Nonlinear regression is an iterative procedure. The program must start with estimated values for each variable that are in the right "ball park"- usually within a factor of five of the actual value. It then adjusts these initial values to improve the fit. It repeats the adjustments until the improvement is no longer significant.

Later sections of this unit explain how to choose initial values for various kinds of experiments. The estimates don't need to be extremely accurate. Nonlinear regression will usually work fine as long as the estimates are within 3 to 5 times their actual values.

Some programs (including GraphPad Prism) automatically choose initial values for you.

\section{Fix one or more variables to a constant value?}

In some situations it makes sense to fix some of the variables to constant values. For example, when analyzing specific (rather than total) binding, the bottom plateau of a dissociation experiment should be defined as a constant equal to zero.

\section{Weighting}

In general, the goal of nonlinear regression is to find the values of the variables in the model that make the curve come as close as possible to the data points. Usually this is done by minimizing the sum of the squares of the vertical distances of the data points from the curve. This is appropriate when the scatter of points around the curve is expected to be Gaussian and unrelated to the $y$ values of the points.

With many experimental protocols, the experimental scatter is not expected to be the same for all points. Instead, the experimental scatter is expected to be a constant percentage of the $y$ value. If this is the case, points with high $y$ values will have more scatter than points with low $y$ values. When the program minimizes the sum of squares, points with high $y$ values will have a larger influence while points with smaller $y$ values will be relatively ignored. This problem may be avoided by minimizing the sum of the square of the relative distances. This procedure is termed weighting the values by $1 / y^{2}$. Because it prevents large points from being over-weighted, the term unweighting seems more intuitive.

Data may be weighted in other ways. The goal is to obtain a measure of goodness-of-fit that weights all the data points equally.

With binding data, scatter is often proportional to the amount of binding, so relative weighting may be appropriate. Results are usually very similar whether or not you choose to use relative weighting. 
If replicate $y$ values are collected at every value of $x$, there are two ways to analyze the data: (1) treat each replicate as a separate point, or (2) average the replicate $y$ values and treat the mean as a single point.

With radioligand binding data, the first approach is usually best, because all the data are obtained from one tissue preparation and the sources of experimental error are independent for each tube. If one value happens to be a bit high, there is no reason to expect the other replicates to be high as well. Each replicate can be considered an independent data point.

Do not treat each replicate as a separate point when the experimental error of the replicates are related. Instead, average the replicates and analyze the averages. This situation doesn't come up often with radioligand binding data, but here is one example. Assume that you perform an experiment with only a single replicate at each value of $y$ (concentration or time) but count each tube three times. It is not fair to enter the three counts as triplicates, and then analyze each triplicate as a separate value. As the replicates are not independent, any experimental error would appear in all the replicates.

\section{Assumptions of Nonlinear Regression}

The results of nonlinear regression are meaningful only if the following assumptions are true (or nearly true):

1. The model is correct. Nonlinear regression adjusts the variables in the equation you chose to minimize the sum of squares. It does not attempt to find a better equation.

2. The variability of values around the curve follow a Gaussian distribution. Even though no biological variable follows a Gaussian distribution exactly, it is sufficient that the variation be approximately Gaussian.

3. The standard deviation (SD) of the residuals is the same everywhere, regardless of the value of $x$. In other words, the average scatter of the points around the curve is the same at all parts of the curve. The assumption is termed homoscedasticity. If the SD is not constant but rather is proportional to the value of $y$, weight the data to minimize the sum of squares of the relative distances.

4. The model assumes that $x$ is known exactly. This is rarely the case, but it is sufficient to assume that any imprecision in measuring $x$ is very small compared to the variability in $y$.

5. The errors are independent. The deviation of each value from the curve should be random, and should not be correlated with the deviation of the previous or next point. If there is any carryover from one sample to the next, this assumption will be violated.

\section{EVALUATING RESULTS OF NONLINEAR REGRESSION}

Before accepting the results of nonlinear regression, the following questions should be asked:

\section{Did the Program Converge on a Solution?}

A nonlinear regression program will stop its iterations when it can't improve the fit by adjusting to the values of any of the variables. At that point, the program is said to have converged on the best fit. In some cases, the program gets stuck. It doesn't know whether the fit would improve by increasing or decreasing the value of a variable. When this happens, the program stops. The exact wording of the error message is unlikely to be 
helpful. In this situation, some programs may still apparently show results, but these "results" do not represent a best-fit curve.

\section{Are the Results Scientifically Plausible?}

The mathematics of curve fitting sometimes yields results that make no scientific sense. For example, noisy or incomplete data can lead to negative rate constants, fractions greater than 1.0 , and negative $K_{\mathrm{d}}$ values.

If the results make no scientific sense, they are unacceptable, regardless of $R^{2}$ and of how close the curve comes to the points. Try a simpler equation, or try fixing some variables to constant values.

Also check that the best-fit values of the variables are reasonable compared to the range of the data. Don't trust the results if the top plateau of a sigmoid curve is far higher than the highest data point. Don't trust the results if an $\mathrm{EC}_{50}$ value is not within the range of the $y$ values.

\section{Does the Curve Come Close to the Points?}

In rare cases, the fit may be far from the data points. This may happen, for example, if the wrong equation is chosen. Look at the graph to make sure this didn't happen.

Goodness of fit can also be evaluated by looking at the value of $R^{2}$ (known by statisticians as the coefficient of determination). $R^{2}$ is the fraction of the total variance of $y$ that is explained by the model (equation). Mathematically, it is defined by the equation: $R^{2}=1.0$ $-\mathrm{SS} / s_{y}{ }^{2}$, where $s_{y}{ }^{2}$ is the variance (standard deviation squared) of $y$ values. The value of $R^{2}$ is always between 0.0 and 1.0 , and it has no units.

When $R^{2}$ equals 0.0 , the best-fit curve fits the data no better than a horizontal line going through the mean of all $y$ values. In this case, knowing $x$ does not help you predict $y$. When $R^{2}=1.0$, all points lie exactly on the curve with no scatter; if $x$ is known, $y$ may be calculated exactly.

If $R^{2}$ is high, the curve comes closer to the points than would a horizontal line through the mean $y$ value, but a high $R^{2}$ should not be overinterpreted. It does not mean that the chosen equation is the best to describe the data. It also does not mean that the fit is unique - other values of the variables may generate a curve that fits just as well.

When comparing one- and two-site models, it is not sufficient to simply compare $R^{2}$ values.

\section{Do the Data Systematically Deviate from the Curve?}

If the data really follow the model described by the chosen equation, the data points should be randomly scattered above and below the curve. The distance of the points from the curve should also be random, and not be related to the value of $x$.

The best way to look for systematic deviations of the points from the curve is to inspect a graph of the residuals and to look at the runs test.

\section{Residuals}

A residual is the distance of a point from the curve. A residual is positive when the point is above the curve, and is negative when the point is below the curve. The residual table

Analyzing Radioligand Binding Data has the same $x$ values as the original data, but each $y$ value is replaced by the vertical distance of the point from the curve. An example is shown in Figure 7.5.24. As shown in 

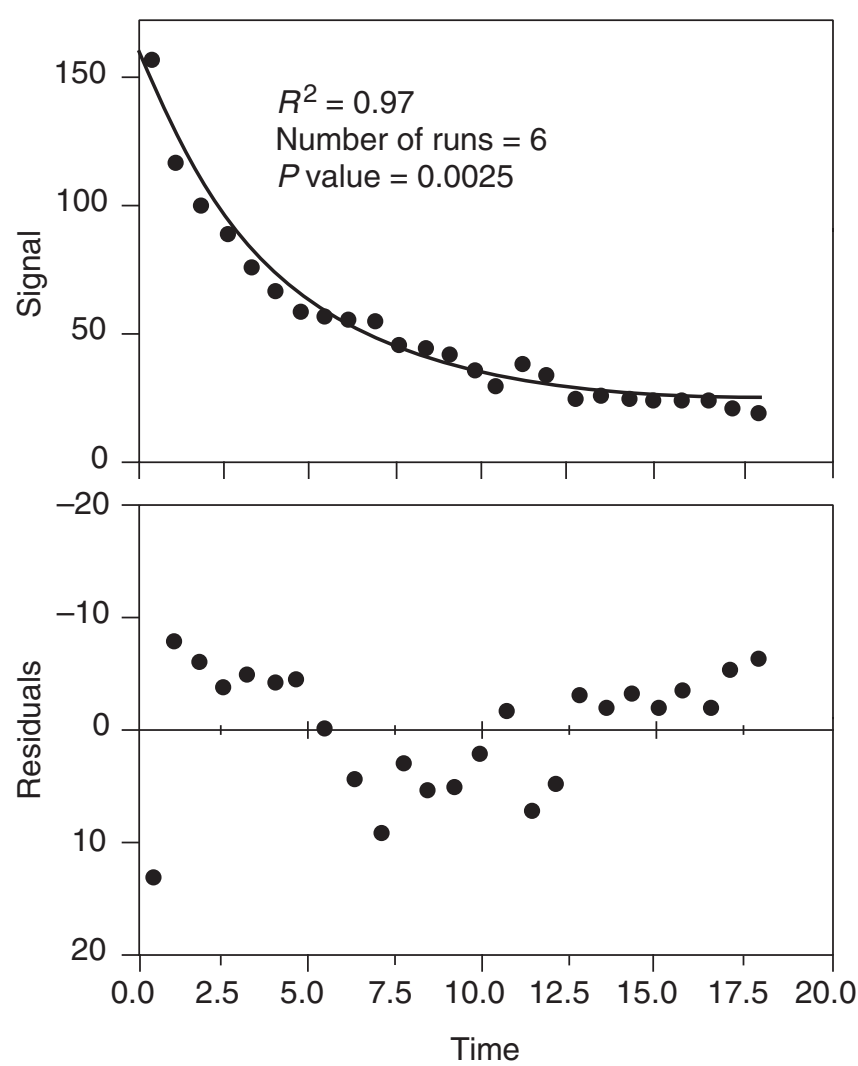

Figure 7.5.24 Residuals. The top panel graphs dissociation kinetic data. The bottom panel shows the residuals (i.e., the $y$ axis plots the distance between the point and the curve from the top panel).

panel A, the data points are not randomly distributed above and below the curve. There are clusters of points all above or all below. This is much easier to see on the graph of the in panel B. The points are not randomly scattered above and below the $x$ axis.

\section{The runs test}

The runs test determines whether the data deviate systematically from the equation you selected. A run is a series of consecutive points that are either all above or all below the regression curve. Another way of saying this is that a run is a series of points whose residuals are either all positive or all negative.

If the data points are randomly distributed above and below the regression curve, it is possible to calculate the expected number of runs. If there are fewer runs than expected, it may mean that the regression model is wrong. If the data really follow the equation used to create the curve, the $P$ value from the runs test may be used to determine the chance of obtaining as few (or fewer) runs as observed in the experiment. If the $P$ value is small, it indicates that the data really don't follow the model.

In the example in Fig. 7.5.24, the equation does not adequately match the data. There are only six runs, and the $\mathrm{P}$ value for the runs test is very small. This means that the data systematically deviate from the curve, and the data were fit to the wrong equation. 


\section{Are the Confidence Intervals Wide?}

In addition to reporting the values of the variables that make the equation fit the data best, nonlinear regression programs also express the uncertainty as a standard error for each variable. Use the standard error to calculate a 95\% confidence interval (CI), if the nonlinear regression program doesn't calculate one. The $95 \%$ confidence interval extends from approximately two standard errors below the best-fit value to approximately two standard errors above the best-fit value. (The number 2.0 is approximate. The exact multiplier comes from the $t$ distribution and depends on the number of degrees of freedom which equals the number of data points minus the number of variables fit by the program.)

The CI means that if all the assumptions of nonlinear regression are true, there is a $95 \%$ chance that the interval contains the true value. More precisely, if a nonlinear regression is performed many times (on different data sets) the expected confidence interval will include the true value $95 \%$ of the time, but exclude the true value the other $5 \%$ of the time.

Three factors can make the confidence interval too narrow:

1. The CI is based only on the scatter of data points around the curve within this one experiment. If the experiment is repeated many times, the scatter between the results is likely to be greater than predicted from the CI determined in one experiment.

2. If any of the assumptions of nonlinear regression are violated, the confidence intervals will probably be too narrow.

3. The confidence intervals from nonlinear regression are calculated using mathematical shortcuts and so are referred to as asymptotic confidence intervals or approximate confidence intervals. In some cases these intervals can be too narrow (too optimistic).

Because of these problems, the confidence intervals should not be interpreted too rigorously. Rather than focusing on the CI reported from analysis of a single experiment, repeat the experiment several times.

If the confidence interval is extremely wide, do not trust the results. Confidence intervals are wide when the data are very scattered or data have not been collected over a wide enough range of $x$ values.

The data in Figure 7.5.25 were fit to a dose-response curve, and the 95\% CI for the $\mathrm{EC}_{50}$ extends over six orders of magnitude. The explanation is simple. Since the data do not define plateaus at either the top or the bottom, zero and one hundred are not defined. This makes it impossible to determine the $\mathrm{EC}_{50}$ with precision.

In this example, it might make scientific sense to set the bottom plateau to $0 \%$ and the top plateau to $100 \%$ (if the plateaus were defined by other controls not shown on the graph). If this were done, the equation would fit fine and the confidence interval would be narrow.

Note that the problem with the fit is not obvious by inspecting a graph, because the curve goes very close to the points. The value of $R^{2}(0.9999)$ is also not helpful. That value also indicates that the curve comes close to the points, but does not indicate whether the fit is unique.

The CI is also wide when data in an important part of the curve has not been collected. The dose-response curve in Figure 7.5.26 has wide confidence intervals. Even when constraining the bottom to be zero and the top to be 100 and the slope to equal 1.0, the

Analyzing Radioligand Binding Data $95 \% \mathrm{CI}$ for the $\mathrm{EC}_{50}$ extends over almost an order of magnitude. The problem is simple. 
The $\mathrm{EC}_{50}$ is the concentration at which the response is half-maximal, and this example has no data near that point.

Finally, the CI is wide if one tries to fit data to a two-site model when the data really follow a one-site model. In this case, the program might report very wide confidence intervals, as it will report that the two sites are very similar.

\section{Is the Fit a Local Minimum?}

The nonlinear regression procedure adjusts the variables in small steps in order to improve the goodness-of-fit. If Prism converges on an answer, altering any of the variables a little bit will make the fit worse. But it is theoretically possible that large changes in the

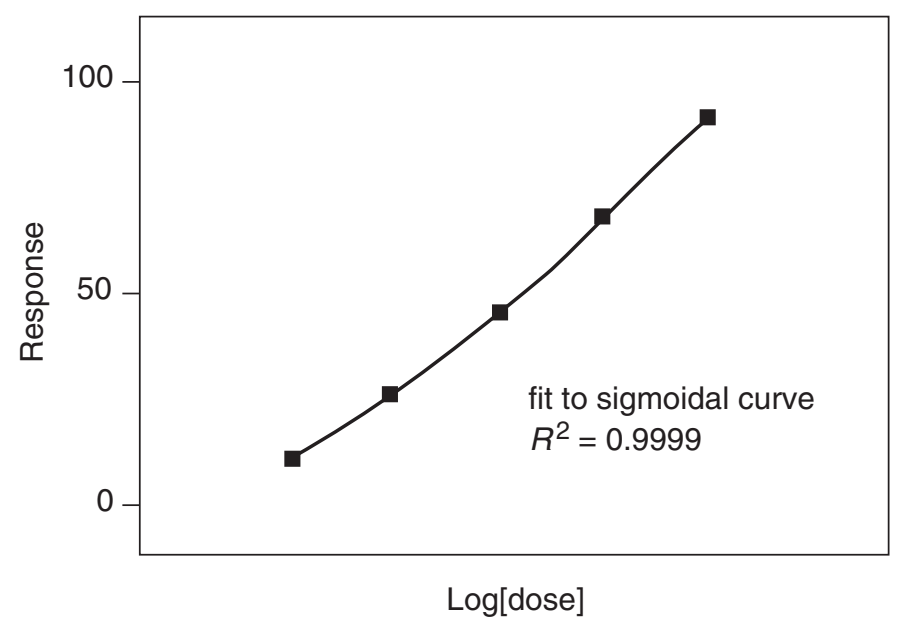

Figure 7.5.25 A dose-response curve with data collected over a narrow range of concentrations. When a nonlinear regression program tries to fit the top and bottom plateaus as well as the $\mathrm{EC}_{50}$ and slope, the resulting confidence intervals are very wide. Since there is no data to define zero and one hundred, the program will be very uncertain about the $\mathrm{EC}_{50}$. If the nonlinear regression program is told to set the top and bottom plateaus to constant values (from controls), then it can determine the $\mathrm{EC}_{50}$ with precision.

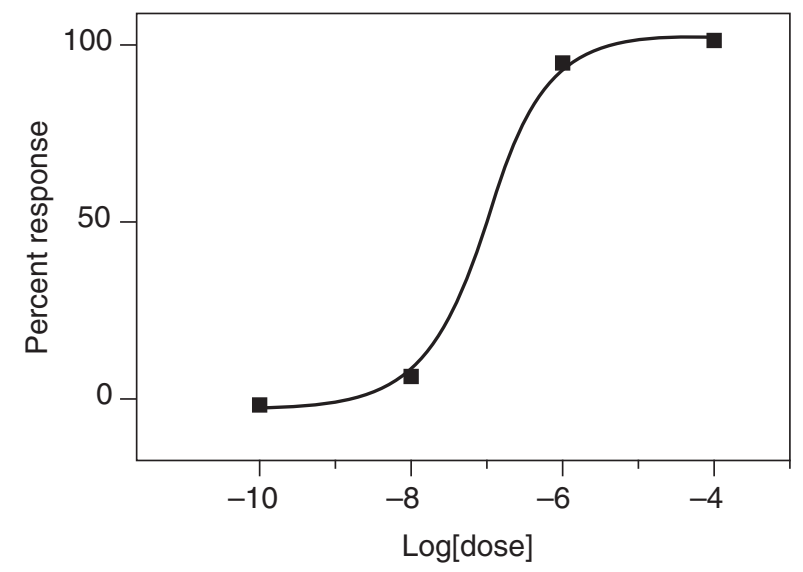

Figure 7.5.26 A dose response curve with no data in the middle of the curve. Since there are no data points in the middle of the curve, the best-fit value of the $\mathrm{EC}_{50}$ will be uncertain with a wide confidence interval.

Neurochemistry/ Neuropharmacology 


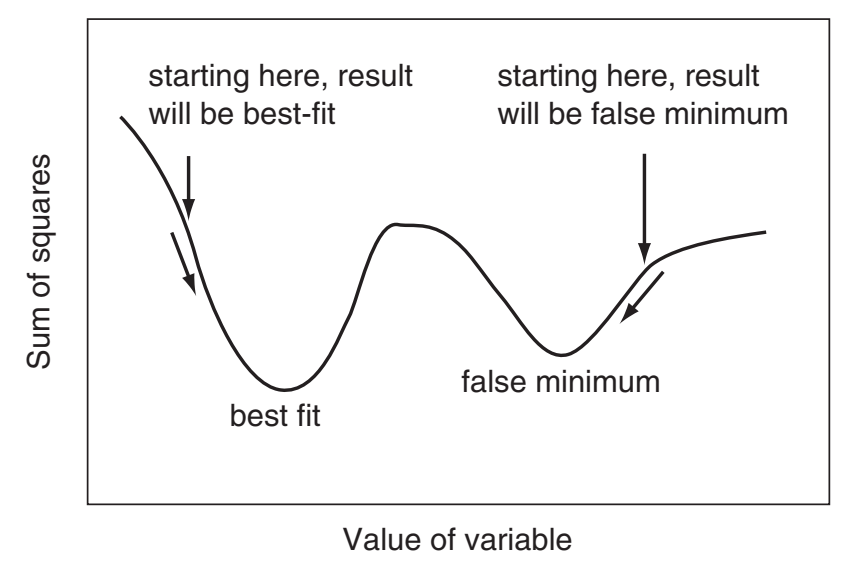

Figure 7.5.27 What is a false minimum? A nonlinear regression program stops when making any small change to a variable will worsen the fit and thus raise the sum of squares. In rare cases, this may happen at a false minimum rather than the true best fit value.

variables might lead to much better goodness-of-fit. Thus, the curve that Prism decides is the "best" may really not be the best.

Think of latitude and longitude as representing two variables Prism is trying to fit. Now think of altitude as the sum of squares. Nonlinear regression works iteratively to reduce the sum of squares. This is like walking downhill to find the bottom of the valley. When nonlinear regression has converged, changing any variable increases the sum of squares. When at the bottom of the valley, every direction leads uphill. But there may be a much deeper valley over the ridge that is unknown (see Fig. 7.5.27). In nonlinear regression, large changes in variables might decrease the sum of squares.

This problem (called finding a local minimum) is intrinsic to nonlinear regression, no matter what program is used. A local minimum will rarely be encountered if the data have little scatter, data is collected over an appropriate range of $x$ values, and an appropriate equation is chosen.

To continue the analogy, the confidence intervals for the variables are very wide when the bottom of the valley is very flat. A great distance can be traveled without changing elevation. The values of the variables can be changed a great deal without changing the goodness-of-fit.

To test for the presence of a false minimum:

1. Note the values of the variables and the sum of squares from the first fit.

2. Make a large change to the initial values of one or more variables and run the fit again. Repeat several times.

3. Ideally, Prism will report nearly the same sum of squares and same variables regardless of the initial values. If the values are different, accept the ones with the lowest sum of squares. 


\section{What to Do When the Fit Is No Good?}

The previous sections explained how to identify a bad fit. If any of these situations are encountered, Table 7.5.9 describes some things to try.

\section{COMPARING TREATMENT GROUPS}

The results of radioligand binding experiments will often be compared between treatment groups. There are three ways to do this.

\section{Compare the Results of Repeated Experiments (Method 1)}

After repeating the experiment several times, compare the best-fit value of a variable in control and treated preparations using a paired $t$ test (or the analogous Wilcoxon nonparametric test).

For example, in Table 7.5.10, the $\log \left(K_{\mathrm{i}}\right)$ values of results from a competitive binding curve performed in two groups of cells are shown. Compare the results using a paired $t$ test. The $t$ ratio is 16.7, and the $P$ value is 0.0036 (two-tail). If the treatment did not alter the $\log \left(K_{\mathrm{i}}\right)$, there is only a $0.36 \%$ chance that such a large difference (or larger) between $\log \left(K_{\mathrm{i}}\right)$ is by chance. Since the $P$ value is so low, conclude that the change in $K_{\mathrm{i}}$ was statistically significant.

Note that we compare $\log \left(K_{\mathrm{i}}\right)$ values rather than $K_{\mathrm{i}}$ values. When doing a paired $t$ test, a key assumption is that the distribution of differences (treated vs. control) follow a Gaussian distribution. Since a competitive binding curve (similar to a dose response curve) is conducted with $x$ values (concentration) equally spaced on a log scale, the uncertainty of the $\mathrm{EC}_{50}$ is reasonably symmetrical (and perhaps Gaussian) when expressed on a $\log$ scale. It is equally likely that the best-fit value of the $\log \left(K_{\mathrm{i}}\right)$ is $0.1 \log$ units too high or $0.1 \log$ units too low. In contrast, the uncertainty in $K_{\mathrm{i}}$ is not symmetrical.

Table 7.5.9 Troubleshooting Guide to Evaluating Results of Nonlinear Regression

\begin{tabular}{ll}
\hline Potential problem & Solution \\
\hline $\begin{array}{l}\text { The equation simply does not describe } \\
\text { the data. }\end{array}$ & Try a different equation. \\
$\begin{array}{l}\text { The initial values are too far from their } \\
\text { correct values. }\end{array}$ & $\begin{array}{l}\text { Enter different initial values. If using a user-defined } \\
\text { equation, check the rules for initial values. }\end{array}$ \\
$\begin{array}{l}\text { The range of } x \text { values is too narrow to } \\
\text { define the curve completely. }\end{array}$ & $\begin{array}{l}\text { If possible, collect more data. Otherwise, hold one } \\
\text { of the variables to a constant value. }\end{array}$ \\
$\begin{array}{l}\text { There is not enough data collected in a } \\
\text { critical range of } x \text { values. }\end{array}$ & $\begin{array}{l}\text { Collect more data in the important regions. } \\
\begin{array}{l}\text { The data are very scattered and don't } \\
\text { really define a curve. }\end{array}\end{array}$ \\
$\begin{array}{l}\text { Try to collect less scattered data. If combining } \\
\text { several experiments, normalize the data for each } \\
\text { experiment to an internal control. }\end{array}$ \\
$\begin{array}{l}\text { component, but the data don't follow a } \\
\text { multicomponent model. }\end{array}$ & $\begin{array}{l}\text { Use a simpler equation. } \\
\text { The numbers are too large. }\end{array}$ \\
$\begin{array}{l}\text { The numbers are too small. } \\
\text { If the } y \text { values are very large, change the units. Do } \\
\text { not use values greater than } \sim 10^{4} .\end{array}$ \\
$\begin{array}{l}\text { If your } y \text { values are very small, change the units. Do } \\
\text { not use values less than } \sim 10^{-4} \text {. }\end{array}$
\end{tabular}

Neurochemistry/ Neuropharmacology

7.5.47 
Table 7.5.10 $\log \left(K_{\mathrm{i}}\right)$ Values for a Sample Competitive Binding Experiment

\begin{tabular}{lll}
\hline Experiment & Control & Treated \\
\hline 1 & -6.13 & -6.53 \\
2 & -6.39 & -6.86 \\
3 & -5.92 & -6.31 \\
\hline
\end{tabular}

\section{Compare the Results within One Experiment: Simple Approach (Method 2)}

Use a $t$ test to determine whether the difference between best-fit values is greater than would be expected by chance, given the standard errors of the variables.

For example, competitive binding curves of control and treated data were compared in an experiment performed once. Nonlinear regression fit three variables, Top, Bottom, and $\log \left(\mathrm{EC}_{50}\right)$. Only the $\log \left(\mathrm{EC}_{50}\right)$ values are of interest. In this example, the control $\log \left(\mathrm{EC}_{50}\right)$ was -6.08 with a standard error of 0.3667 . The treated $\log \left(\mathrm{EC}_{50}\right)$ was -6.20 with a standard error of 0.0617 .

Compare the two groups with an unpaired $t$ test.

1. Calculate the $t$ ratio as the difference between $\log \left(\mathrm{EC}_{50}\right)$ values divided by the standard error of that difference (calculated from the two standard errors). Since the sample size is the same in the two groups, use the equation:

$$
t=\frac{\log \left(\mathrm{EC}_{50}\right)_{\mathrm{A}}-\log \left(\mathrm{EC}_{50}\right)_{\mathrm{B}}}{\sqrt{\mathrm{SEM}_{\mathrm{A}}^{2}+\mathrm{SEM}_{\mathrm{B}}^{2}}}=2.292
$$

2. Calculate the number of degrees of freedom (DF), which equals the sum of the number of degrees of freedom in each group. This equals the number of data points minus the number of variables fit by the nonlinear regression procedure. In this example, there were 15 data points, and three variables were fit. So there are $12 \mathrm{DF}$ in each group, and $24 \mathrm{DF}$ altogether.

3. Use a table or program to determine a $P$ value that corresponds to the values of $t$ and DF. For this example, the $P$ value is 0.0309 . If the treatment really didn't alter the $\mathrm{EC}_{50}$, there is only a $3.09 \%$ chance that this large of a difference (or more) is by coincidence. Since the $P$ value is so low, it is concluded that the two $\mathrm{EC}_{50}$ values are statistically significantly different.

GraphPad Prism, GraphPad InStat and many other programs can compute $t$ and the $P$ value from data entered as mean, SEM, and $N$. Enter the best-fit value of the $\log \left(\mathrm{EC}_{50}\right)$ (or any other fit variable) instead of the mean, and the SE of that variable instead of the SEM. The trick is figuring out what value to enter as " $N$ " (sample size). Remember that:

1. For nonlinear regression, the number of degrees of freedom equals the number of data points minus the number of variables fit.

2. For an ordinary $t$ test, the number of degrees of freedom for each sample equals one less than the number of data points.

3. The $t$ test calculations are based on the numbers of degrees of freedom. However, most programs ask for $N$ instead and then compute DF as $N-1$. When comparing the results of nonlinear regression, enter $N$ as the number of degrees of freedom plus 1. The program will subtract 1 to determine the DF. All the other calculations are 
based on the value of DF, and $N$ is ignored. In this example, enter $N=12+1=13$ for each group.

This method only uses data from one experiment. The SE value is a measure of how precisely the $\log \left(\mathrm{EC}_{50}\right)$ has been determined in this one experiment. It is not a measure of how reproducible the experiment is. Despite the impressive $P$ value, these results should not be trusted until the experiment is repeated.

The $t$ test assumes that the uncertainty in the values of the variables follows a Gaussian distribution. This assumption is not necessarily true with the SE values that emerge from nonlinear regression. The only way to assess the validity of this assumption is to simulate many sets of data, fit each with nonlinear regression, and examine the distribution of best-fit values. This has been done with many commonly used equations, and it seems that the assumption is reasonable in many cases.

Compare $\log \left(\mathrm{EC}_{50}\right)$, not $\mathrm{EC}_{50}$. You want to express the variables in a form that makes the uncertainty as symmetrical and Gaussian as possible. Since a competitive binding curve (similar to a dose response curve) is conducted with $x$ values (concentration) equally spaced on a $\log$ scale, the uncertainty of $\log \left(\mathrm{EC}_{50}\right)$ is reasonably symmetrical (and perhaps Gaussian). It is equally likely that the observed $\log \left(K_{\mathrm{i}}\right)$ is $0.1 \log$ units too high or $0.1 \log$ units too low. In contrast, the uncertainty in $K_{\mathrm{i}}$ is not symmetrical.

\section{Compare the Results Within One Experiment: More Complicated Approach (Method 3)}

The method of the previous section only compared the value of the $\log \left(\mathrm{EC}_{50}\right)$. This section describes a more general method to compare entire curves to ask whether the data sets differ at all. The idea is to first fit the two curves separately, and then combine the values and fit one curve to all the data.

Follow these steps:

1. Fit the two data sets separately as in the previous section.

2. Total the sum of squares and DF from the two fits. For this example the total sum of squares equals $19,560+29,320=48,880$, and the total DF equals $12+12=24$. Since these are the results of fitting the two data sets separately, label these values $\mathrm{SS}_{\text {separate }}$ and $\mathrm{DF}_{\text {separate. }}$.

3. Combine the two data sets into one. For this example, the combined data set has 30 $x y$ pairs, with each $x$ value appearing twice.

4. Fit the combined data set to the same equation. Note the SS and DF. For this example, $\mathrm{SS}=165,200$, and $\mathrm{DF}=27$ (30 data points minus three variables). Call these values $\mathrm{SS}_{\text {combined }}$ and $\mathrm{DF}_{\text {combined }}$.

5. $\mathrm{SS}_{\text {separate }}$ is expected to be smaller than $\mathrm{SS}_{\text {combined }}$ even if the curves are really identical, simply because the separate fits have more degrees of freedom. The question is whether the SS values are more different than expected by chance. To find out, calculate the $F$ ratio using the equation:

$$
F=\left(\frac{\mathrm{SS}_{\text {combined }}-\mathrm{SS}_{\text {separate }}}{\mathrm{SS}_{\text {combined }}}\right) /\left(\frac{\mathrm{DF}_{\text {combined }}-\mathrm{DF}_{\text {separate }}}{\mathrm{DF}_{\text {separate }}}\right)
$$

For this example, $F=19.03$.

Neurochemistry/

Neuropharmacology

7.5.49 
6. Determine the $P$ value from $F$. There are $\mathrm{DF}_{\text {combined }}-\mathrm{DF}_{\text {separate }}$ degrees of freedom in the numerator, and $\mathrm{DF}_{\text {separate }}$ degrees of freedom in the denominator. GraphPad StatMate can calculate the $P$ value (from $F$ and the two DF values), or it may be found in the back of most statistics books.

7. For this example, the $P$ value is $<0.0001$. If the treatment were really ineffective, there is less than a $0.01 \%$ chance that the two curves would differ as much (or more) as they differed in this experiment. Since the $P$ value is low, you'll conclude that the curves are really different.

This method only uses data from one experiment. Despite the impressive $P$ value, these results should not be trusted until the experiment is repeated. This method compares the curves overall. It doesn't determine which variable(s) are different. Differences might be due to something trivial such as a different baseline, rather than something important such as a different $\mathrm{EC}_{50}$.

\section{Advantages and Disadvantages of the Three Approaches}

If the experiment has been repeated several times, use the first method. There are two advantages. The first is that compared to the other methods discussed below, this method is far easier to understand and communicate to others. Second, the entire test is based on the consistency of the results between repeat experiments. Since there are usually more causes for variability between experiments than within experiments, it makes sense to base the comparison on differences between experiments.

The disadvantage of the first method is that information is being discarded. The calculations are based only on the best-fit value from each experiment, and they ignore the SE of those values presented by the curve fitting program.

If the experiment has been performed only once, the experiment should be repeated. Regardless of what statistical results are obtained, results from a single experiment should not be trusted. To compare results in a single experiment, use Method 2 or 3.

Generally only one variable is of interest (i.e., a rate constant or $\mathrm{EC}_{50}$ ); the others are less important. Method 2 compares the variable of interest. Method 3 is more general. Since the method compares the entire curve, it does not force a decision regarding which variable(s) to compare. This is both its advantage and disadvantage.

\section{CALCULATIONS WITH RADIOACTIVITY}

\section{Efficiency of Detecting Radioactivity}

Efficiency is the fraction of radioactive disintegration that is detected by the counter. Efficiency is determined by counting a standard sample under conditions identical to those used in the experiment.

With ${ }^{125} \mathrm{I}$, the efficiency is usually $>90 \%$, depending on the geometry of the counter. The efficiency is not $100 \%$ because the detector doesn't entirely surround the tube, which allows a few gamma rays (photons) to miss the detector.

With ${ }^{3} \mathrm{H}$, the efficiency of counting is much lower, and usually varies between $40 \%$ and $50 \%$. The low efficiency is mostly a consequence of the physics of decay and cannot be improved by better instrumentation or better scintillation fluid. When a tritium atom decays, a neutron converts to a proton and the reaction emits an electron and neutrino. The energy released is always the same, but it is randomly partitioned between the neutrino (not detected) and an electron (detection attempted). When the electron has 
sufficient energy, it will travel far enough to encounter a fluor molecule in the scintillation fluid. This fluid amplifies the signal and gives off a flash of light detected by the scintillation counter. The intensity of the flash (number of photons) is proportional to the energy of the electron. If the electron has insufficient energy, it is not captured by the fluor and is not detected. If it has low energy, it is captured but the light flash has few photons and is not detected by the instrument. Since the decay of many tritium atoms does not lead to a detectable number of photons, the efficiency of counting is much less than $100 \%$.

Efficiency of counting ${ }^{3} \mathrm{H}$ is reduced by the presence of any color in the counting tubes, if the mixture of water and scintillation fluid is not homogeneous, or if the radioactivity is trapped in tissue (so emitted electrons don't travel into the scintillation fluid).

\section{Specific Radioactivity}

Radioligand packaging usually states the specific radioactivity as Curies per millimole $(\mathrm{Ci} / \mathrm{mmol})$. Because measurements are expressed in counts per minute $(\mathrm{cpm})$, the specific radioactivity is more useful when stated in $\mathrm{cpm}$. Often the specific radioactivity is expressed as $\mathrm{cpm} / \mathrm{fmol}\left(1 \mathrm{fmol}=10^{-15} \mathrm{~mole}\right)$.

To convert from $\mathrm{Ci} / \mathrm{mmol}$ to $\mathrm{cpm} / \mathrm{fmol}$, know that $1 \mathrm{Ci}$ equals $2.22 \times 10^{12}$ disintegrations per minute (dpm). Use this equation to convert $Z \mathrm{Ci} / \mathrm{mmol}$ to $Y \mathrm{cpm} / \mathrm{fmol}$ when the counter has an efficiency (expressed as a fraction) equal to $E$.

$$
\begin{aligned}
& Y \frac{\mathrm{cpm}}{\mathrm{fmol}}=Z \frac{\mathrm{Ci}}{\mathrm{mmol}} \times 2.22 \times 10^{12} \frac{\mathrm{dpm}}{\mathrm{Ci}} \times 10^{-12} \frac{\mathrm{mmol}}{\mathrm{fmol}} \times E \frac{\mathrm{cpm}}{\mathrm{dpm}} \\
& Y=Z \times 2.22 \times E \quad(\text { in cpm } / \mathrm{fmol})
\end{aligned}
$$

For example, the specific activity will be $2190 \mathrm{Ci} / \mathrm{mmol}$ if every molecule incorporates exactly one ${ }^{125} \mathrm{I}$ atom. If the counting efficiency is $85 \%$, then the specific activity is 2190 $\times 2.22 \times 0.85=4133 \mathrm{cpm} / \mathrm{fmol}$.

In many countries, radioligand packaging states the specific radioactivity in $\mathrm{GBq} / \mathrm{mmol}$, rather than $\mathrm{Ci} / \mathrm{mmol}$. To convert to $\mathrm{cpm} / \mathrm{fmol}$, you need to know that $1 \mathrm{~Bq}$ (Becquerel) is one radioactive disintegration per second $\left(1 \mathrm{GBq}=10^{9} \mathrm{dps}\right)$. To convert from $\mathrm{GBq} / \mathrm{mmol}$ to $\mathrm{cpm} / \mathrm{fmol}$, use this equation.

$$
Y \frac{\mathrm{cpm}}{\mathrm{fmol}}=Z \frac{\mathrm{GBq}}{\mathrm{mmol}} \times 10^{9} \frac{\mathrm{dps}}{\mathrm{GBq}} \times 60 \frac{\mathrm{sec}}{\mathrm{min}} \times 10^{-12} \frac{\mathrm{mmol}}{\mathrm{fmol}} \times E \frac{\text { counts }}{\text { disintegrations }}=Z \times 0.06 \times E
$$

If every molecule is labeled with ${ }^{125} \mathrm{I}$, the specific activity is $81,030 \mathrm{GBq} / \mathrm{mmol}$. If the counting efficiency is $85 \%$, then the specific activity can also be expressed as $81,030 \times$ $0.06 \times 0.85=4133 \mathrm{cpm} / \mathrm{fmol}$.

\section{Calculating the Concentration of the Radioligand}

Rather than trust dilutions, the concentration of radioligand in a stock solution can be accurately calculated. Measure the cpm in a small volume of solution and use the following equation, in which $C$ is cpm counted, $V$ is volume of the solution in $\mathrm{ml}$, and $Y$ is the specific activity of the radioligand in $\mathrm{cpm} / \mathrm{fmol}$ (calculated in the previous section).

$$
\text { concentration in } \mathrm{pM}=\frac{C \mathrm{cpm}}{\frac{Y \mathrm{cpm} / \mathrm{fmol}}{V \mathrm{ml}} \times \frac{0.001 \mathrm{pmol} / \mathrm{fmol}}{0.001 \mathrm{liter} / \mathrm{ml}}}=\frac{C / Y}{V}
$$




\section{Radioactive Decay}

Radioactive decay is entirely random. The probability of decay at any particular interval is the same as the probability of decay during any other interval. Starting with $N_{0}$ radioactive atoms, the number remaining at time $t$ is:

$$
N_{t}=N_{0} \times e^{-k_{\text {decay }} t}
$$

The rate constant of decay $\left(k_{\text {decay }}\right)$ is expressed in units of inverse time. Each radioactive isotope has a different value of $k_{\text {decay }}$. The value $e$ refers to the base of natural logarithms (2.71828).

The half-life $\left(t_{1}\right)$ is the time it takes for half the isotope to decay. Half-life and the decay rate constant are related by this equation:

$$
t_{1 / 2}=\frac{\ln (2)}{k_{\text {decay }}}=\frac{0.693}{k_{\text {decay }}}
$$

Table 7.5.11 shows the half-lives and rate constants for commonly used radioisotopes. The table also shows the specific activity assuming that each molecule is labeled with one radioactive atom. (This is often the case with ${ }^{125} \mathrm{I}$ and ${ }^{32} \mathrm{P}$. Tritiated molecules often incorporate two or three tritium atoms, which increases the specific radioactivity.)

Radioactive decay can be calculated from a date where you knew the concentration and specific radioactivity using this equation.

$$
\text { fraction remaining }=e^{-k_{\text {decay }} t}
$$

For example, after ${ }^{125} \mathrm{I}$ decays for 20 days, the fraction remaining equals $79.5 \%$. Although data appear to be scanty, most scientists assume that the energy released during decay destroys the ligand so it no longer binds to receptors. Therefore the specific radioactivity does not change over time. What changes is the concentration of ligand. After 20 days, the concentration of the iodinated ligand is $79.5 \%$ of what it was originally, but the specific radioactivity remains $2190 \mathrm{Ci} / \mathrm{mmol}$. This approach assumes that the unlabeled decay product is not able to bind to receptors and has no effect on the binding. Rather than trust this assumption, use newly synthesized or repurified radioligand for key experiments.

Calculations of radioactive decay are straightforward only when each molecule is labeled with a single radioactive isotope, as is usually the case. If a molecule is labeled with several radioactive isotopes, the effective half-life is shorter. If only a fraction of the molecules are labeled with a radioactive isotope, then the decay formula only applies to the labeled portion of the mixture, as the concentration of the unlabeled compound never changes.

Table 7.5.11 Half-Lives and Rate Constants for Commonly Used Isotopes

\begin{tabular}{llll}
\hline Isotope & Half-life & $k_{\text {decay }}$ & $\begin{array}{l}\text { Specific } \\
\text { radioactivity }\end{array}$ \\
\hline${ }^{3} \mathrm{H}$ & 12.43 years & $0.056 \mathrm{year}^{-1}$ & $28.7 \mathrm{Ci} / \mathrm{mmol}$ \\
${ }^{125} \mathrm{I}$ & 59.6 days & $0.0116 \mathrm{day}^{-1}$ & $2190 \mathrm{Ci} / \mathrm{mmol}$ \\
${ }^{32} \mathrm{P}$ & 14.3 days & $0.0485 \mathrm{day}^{-1}$ & $9128 \mathrm{Ci} / \mathrm{mmol}$ \\
${ }^{35} \mathrm{~S}$ & 87.4 days & $0.0079 \mathrm{day}^{-1}$ & $1493 \mathrm{Ci} / \mathrm{mmol}$ \\
\hline
\end{tabular}




\section{Counting Error and the Poisson Distribution}

The decay of a population of radioactive atoms is random, and therefore subject to a sampling error. For example, the radioactive atoms in a tube containing $1000 \mathrm{cpm}$ of radioactivity won't give off exactly 1000 counts in every minute. There will be more counts in some minutes and fewer in others, with the distribution of counts following a Poisson distribution. This variability is intrinsic to radioactive decay and cannot be reduced by more careful experimental controls. There is no way to know the "real" number of counts, but a range of counts can be calculated that is $95 \%$ certain to contain the true average value. As long as the number of counts $(C)$ is greater than $\sim 100$, the confidence interval can be calculated using this approximation:

$$
95 \% \text { CI: }(C-1.96 \sqrt{C}) \text { to }(C+1.96 \sqrt{C})
$$

Computer programs can calculate a more exact confidence interval, as becomes necessary when $C$ is less than $\sim 100$. For example, if $\mathrm{C}=100$, the simple equation above calculates a $95 \%$ confidence interval from approximately 80 to 120 . A more exact equation calculates an interval from 81.37 to 121.61 .

When calculating the confidence interval, set $C$ equal to the total number of counts you measured experimentally, not the number of counts per minute.

For example, if a radioactive sample is placed into a scintillation counter for $10 \mathrm{~min}$, the counter detects 225 counts per minute. What is the $95 \%$ confidence interval? Since the total time was $10 \mathrm{~min}$, the instrument must have detected 2250 radioactive disintegrations. The $95 \%$ confidence interval of this number extends from 2157 to 2343 . This is the confidence interval for the number of counts in $10 \mathrm{~min}$, so the $95 \%$ confidence interval for the average number of counts per minute extends from 216 to 234 . That is, there is a $95 \%$ certainty that the average cpm value lies within this range.

The Poisson distribution explains why it is helpful to count samples longer when the number of counts is small. For example, Table 7.5.12 shows the confidence interval for $100 \mathrm{cpm}$ counted for various times. When longer times are used, the confidence interval is narrower.

Table 7.5.12 Determination of Confidence Values

\begin{tabular}{lccc}
\hline & $1 \mathrm{~min}$ & $10 \mathrm{~min}$ & $100 \mathrm{~min}$ \\
\hline Counts per min $(\mathrm{cpm})$ & 100 & 100 & 100 \\
Total counts & 100 & 1000 & 10000 \\
$95 \%$ CI of counts & 81.4 to 121.6 & 938 to 1062 & 9804 to 10196 \\
$95 \%$ CI of cpm & 81.4 to 121.6 & 93.8 to 106.2 & 98.0 to 102.0 \\
\hline
\end{tabular}

Figure 7.5.28 shows percent error as a function of $C$. Percent error is defined from the width of the confidence interval divided by the number of counts. Of course this graph only shows error due to the randomness of radioactive decay. This is only one source of error in most experiments. 


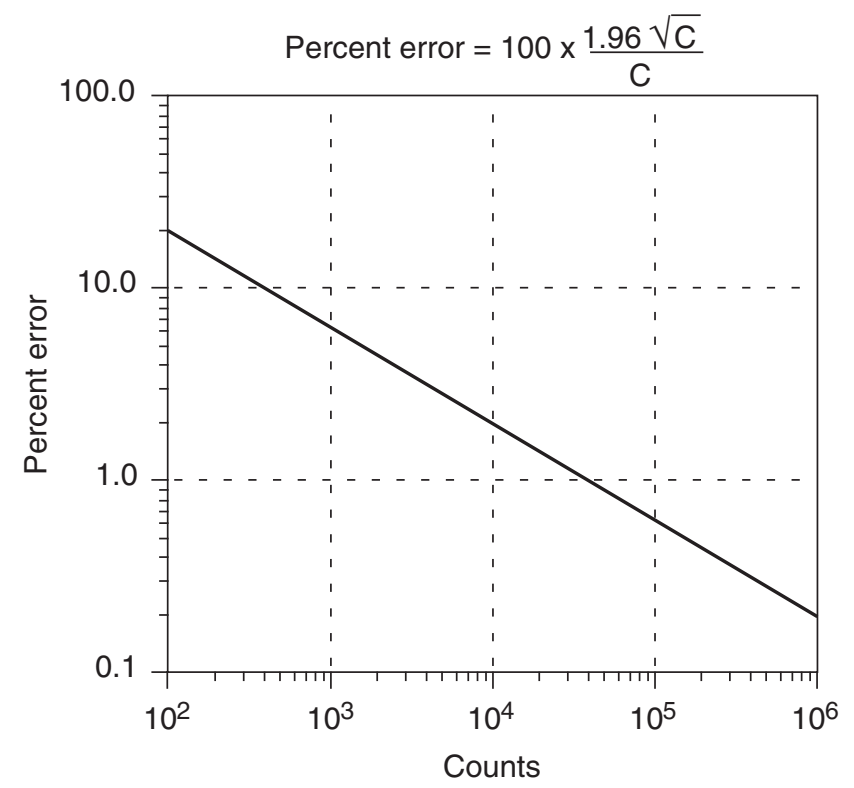

Figure 7.5.28 Counting error. With more counts, the fractional counting error decreases. The $x$ axis shows the number of radioactive decays actually counted (counts per minute times number of minutes).

\section{ANALYZING DATA WITH GRAPHPAD PRISM}

GraphPad Prism is a general purpose program for scientific graphics, statistics and nonlinear regression, available for both Windows and Macintosh computers.

While Prism is not designed especially for analyses of binding data, it is very well suited for analyses. It provides a menu of commonly used equations, including all equations listed in this unit, and can automatically compare one- and two-site models with an $F$ test. When analyzing competitive binding curves, Prism calculates the $K_{\mathrm{i}}$ from the $\mathrm{IC}_{50}$. The program can automatically create a residual plot and calculate the runs test. In addition, Prism's manual and help screens, like this unit, explain the principles of curve fitting.

A trial version of the Windows or Mac versions of Prism can be obtained from the GraphPad web site at http://www.graphpad.com. The trial software is also available on the CD-ROM version of Current Protocols in Neurosciences. The trial versions let you analyze data for an unlimited period of time. For the first thirty days, the Windows version is fully functional. After that, data may be analyzed, but the ability to to print, save, and export will be disabled.

Contact GraphPad Software (SUPPLIERS APPENDIX) to obtain a brochure and trial disk, or to ask questions. 


\section{LITERATURE CITED}

Cheng, Y. and Prusoff, W.H. 1973. Relationship between the inhibition constant $\left(\mathrm{K}_{\mathrm{i}}\right)$ and the concentration of an inhibitor that causes a $50 \%$ inhibition ( $\left.\mathrm{I}_{50}\right)$ of an enzymatic reaction. Biochem. Pharmacol. 22:3099-3108.

Kenakin, T. 1993. Pharmacologic Analysis of DrugReceptor Interactions. Raven Press, New York.

Limbird, L.E. 1996. Cell Surface Receptors: A Short Course in Theory and Methods, 2nd ed. Kluwer Academic Publishers, Boston.

Motulsky, H.J. and Mahan, L.C. 1984. The kinetics of competitive radioligand binding predicted by the law of mass action. Mol. Pharmacol. 25:1-9.

Munson, P.J. and Rodbard, D. 1980. Ligand: A versatile computerized approach to characterization of ligand binding systems. Anal. Biochem. 107:220-239.
Rosenthal, H.E. 1967. A graphic method for the determination and presentation of binding parameters in complex systems. Anal. Biochem. 20:525-532.

Swillens, S. 1995. Interpretation of binding curves obtained with high receptor concentrations: Practical aid for computer analysis. Mol. Pharmacol. 47:1197-1203.

Contributed by Harvey Motulsky

GraphPad Software

San Diego, California

Richard Neubig

University of Michigan

Ann Arbor, Michigan
Neurochemistry/

Neuropharma-

cology 